TRANSACTIONS OF THE

AMERICAN MATHEMATICAL SOCIETY

Volume 357, Number 3, Pages 851-873

S 0002-9947(04)03766-3

Article electronically published on October 19, 2004

\title{
A QUADRATIC APPROXIMATION TO THE SENDOV RADIUS NEAR THE UNIT CIRCLE
}

\author{
MICHAEL J. MILLER
}

\begin{abstract}
Define $S(n, \beta)$ to be the set of complex polynomials of degree $n \geq 2$ with all roots in the unit disk and at least one root at $\beta$. For a polynomial $P$, define $|P|_{\beta}$ to be the distance between $\beta$ and the closest root of the derivative $P^{\prime}$. Finally, define $r_{n}(\beta)=\sup \left\{|P|_{\beta}: P \in S(n, \beta)\right\}$. In this notation, a conjecture of Bl. Sendov claims that $r_{n}(\beta) \leq 1$.

In this paper we investigate Sendov's conjecture near the unit circle, by computing constants $C_{1}$ and $C_{2}$ (depending only on $n$ ) such that $r_{n}(\beta) \sim$ $1+C_{1}(1-|\beta|)+C_{2}(1-|\beta|)^{2}$ for $|\beta|$ near 1 . We also consider some consequences of this approximation, including a hint of where one might look for a counterexample to Sendov's conjecture.
\end{abstract}

\section{INTRODUCTION}

In 1962, Sendov conjectured that if a polynomial (with complex coefficients) has all its roots in the unit disk, then within one unit of each of its roots lies a root of its derivative. More than 50 papers have been published on this conjecture, but it has been verified in general only for polynomials of degree at most 8 [4].

Let $n \geq 2$ be an integer and let $\beta$ be a complex number of modulus at most 1 . Define $S(n, \beta)$ to be the set of polynomials of degree $n$ with complex coefficients, all roots in the unit disk and at least one root at $\beta$. For a polynomial $P$, define $|P|_{\beta}$ to be the distance between $\beta$ and the closest root of the derivative $P^{\prime}$. Finally, define $r_{n}(\beta)=\sup \left\{|P|_{\beta}: P \in S(n, \beta)\right\}$, and note that $r_{n}(\beta) \leq 2$ (since by the GaussLucas Theorem [5. Theorem 6.1] all roots of each $P^{\prime}$ are also in the unit disk, and so each $\left.|P|_{\beta} \leq 2\right)$. In this notation, Sendov's conjecture claims that $r_{n}(\beta) \leq 1$.

In estimating $r_{n}(\beta)$, we will assume without loss of generality (by rotation) that $0 \leq \beta \leq 1$. It is already known that $r_{2}(\beta)=(1+\beta) / 2$ and that

$$
r_{3}(\beta)=\left[3 \beta+\left(12-3 \beta^{2}\right)^{1 / 2}\right] / 6
$$

[9, Theorem 2], that $r_{n}(0)=(1 / n)^{1 /(n-1)}$ [2, Lemma 4 and $\left.p(z)=z^{n}-z\right]$, that $r_{n}(1)=1$ [10, Theorem 1], and that $r_{n}(\beta) \leq \min (1.08332,1+0.72054 / n)$ [1] Corollary 1 and equation (3)].

Received by the editors October 15, 2001.

2000 Mathematics Subject Classification. Primary 30C15.

Key words and phrases. Sendov, Ilieff, Ilyeff. 
Since $r_{n}(1)=1$, an obvious place to look for counterexamples to Sendov's conjecture is in a neighborhood of $\beta=1$. This has already been done in [7, Theorem 3] and [12], where a linear upper bound on $r_{n}(\beta)$ suffices to verify the Sendov conjecture if $\beta$ is sufficiently close to 1 . Unfortunately, having only an upper bound leaves many interesting questions about the conjecture unanswered. In this paper we investigate Sendov's conjecture much more thoroughly near $\beta=1$, by providing a quadratic approximation to $r_{n}(\beta)$ with

Theorem 1. Let $n \geq 3$, let $k$ be the largest integer such that $k \leq(n+1) / 3$ and let

$$
\begin{aligned}
u_{1} & =\cos \frac{2 \pi k}{n+1}, \quad u_{2}=\cos \frac{2 \pi(k+1)}{n+1}, \\
D_{1} & =\frac{-2 u_{1} u_{2}-1}{2\left(1-u_{1}\right)\left(1-u_{2}\right)}, \quad D_{2}=\frac{-1}{2\left(1-u_{1}\right)\left(1-u_{2}\right)}, \\
D_{3} & =\left(-1-4 D_{1}-3 D_{1}^{2}+2 D_{2}^{2}\right) / 2, \\
D_{4} & =\left(3 D_{1}-4 D_{2}+3 D_{1}^{2}-2 D_{1} D_{2}-6 D_{2}^{2}\right) / 2, \\
D_{5} & =\left(2+4 D_{1}+5 D_{2}+2 D_{1}^{2}+4 D_{1} D_{2}+3 D_{2}^{2}\right) / 2, \\
D_{6} & =\left(2 D_{2}+2 D_{1} D_{2}+3 D_{2}^{2}\right) / 2 \quad \text { and } \\
D & =D_{3} n+D_{4}+D_{5} / n+D_{6} / n^{2} .
\end{aligned}
$$

If $n=3$ or $n=5$, then let $\alpha=3 / 2$; otherwise let $\alpha=2$. If $n=5$, then let $\Delta=7 / 225$; otherwise let $\Delta=0$. Then for $\beta$ sufficiently close to 1 , we have

$$
r_{n+1}(\beta)=1+\left(D_{1}+D_{2} / n\right)(1-\beta)+(D+\Delta)(1-\beta)^{2}+\mathcal{O}(1-\beta)^{\alpha+1} .
$$

Before proving this theorem, we will examine some of its consequences. Our first consequence improves on estimates in [7] and [12] (by providing a value for the coefficient of the linear term) with

Corollary 2. For all $n \geq 2$ we have $r_{n}(\beta) \leq 1-(3 / 10)(1-\beta)+\mathcal{O}(1-\beta)^{2}$.

Proof. Recall that for $2 \leq n \leq 3$ we have formulas for $r_{n}(\beta)$, and so the result for those values of $n$ follows from the Taylor series of these formulas at $\beta=1$. As we will show in part 6 of Lemma 8 the quantity $D_{1}+D_{2} / n \leq-3 / 10$ for all $n \geq 3$, and so the rest of Corollary 2 follows from Theorem 1 .

As we will show in part 6 of Lemma 8 , at $n=4$ we have $D_{1}+D_{2} / n=-3 / 10$, so Corollary 2 provides the smallest possible linear upper bound for $r_{n}(\beta)$ that is independent of $n$.

A second consequence of Theorem 1 1 shows that the result of [7, Theorem 3] is the best possible (in the sense that $1 / 3$ cannot be replaced by a larger number), with

Corollary 3. There exist constants $K_{n}>0$ with $\lim _{n \rightarrow \infty} K_{n}=1 / 3$ such that

$$
r_{n+1}(\beta)=1-K_{n}(1-\beta)+\mathcal{O}(1-\beta)^{2} .
$$

Proof. Choose $K_{n}=-\left(D_{1}+D_{2} / n\right)$ and note that by Theorem 1 we have $r_{n+1}(\beta)=$ $1-K_{n}(1-\beta)+\mathcal{O}(1-\beta)^{2}$. As we shall see in parts 5 and 6 of Lemma 8 , the quantity $D_{1}+D_{2} / n$ is negative and tends to $-1 / 3$. 
Recall that $r_{n}(0)=(1 / n)^{1 /(n-1)}$. This quantity is increasing in $n$, so it is tempting to conjecture that for all fixed $\beta$ the quantity $r_{n}(\beta)$ is increasing in $n$. Indeed, the graphs in [6, figure 4.8] provide some evidence of this for $n=4,6,8$, 10 , and 12. Unfortunately, this conjecture is false, as is shown by

Corollary 4. For $\beta$ sufficiently close to 1 we have $r_{6}(\beta)<r_{4}(\beta)$.

Proof. By Theorem 1 and the constants we will compute at the beginning of section 2 we know that

$$
r_{4}(\beta)=1-(1 / 3)(1-\beta)+\mathcal{O}(1-\beta)^{2}
$$

and that

$$
r_{6}(\beta)=1-(11 / 30)(1-\beta)+\mathcal{O}(1-\beta)^{2},
$$

and the conclusion follows.

Corollary 2 hints of the existence of a better-than-Sendov result, for near $\beta=1$ it appears that $r_{n}(\beta)$ is bounded above by a function that is independent of $n$ and strictly less than one. Unfortunately, moving up to the quadratic approximation in Theorem 1 casts doubt upon such a result. To see this, note that as $n$ goes to infinity, then $k /(n+1)$ tends to $1 / 3$, so $u_{1}$ and $u_{2}$ tend to $-1 / 2$, so $D_{3}$ tends to $4 / 81$ and $D_{4}$ tends to $-1 / 9$, and so $D+\Delta$ tends to infinity. Indeed, for $n$ sufficiently large one might expect $r_{n+1}(\beta)>1$ roughly when $D_{1}(1-\beta)+\left(D_{3} n+D_{4}\right)(1-\beta)^{2}>0$, i.e. when $\beta<1+D_{1} /\left(D_{3} n+D_{4}\right) \sim 1-27 /(4 n-9)$, provided that this $\beta$ is "sufficiently close to 1 ". This is an intriguing possibility that is clearly worthy of further investigation.

We will verify Theorem[1] by proving the following three propositions:

Proposition 5. Assume the notation of Theorem 1, Then for all polynomials $P \in S(n+1, \beta)$, we have

$$
|P|_{\beta} \leq 1+\left(D_{1}+D_{2} / n\right)(1-\beta)+(D+\Delta)(1-\beta)^{2}+\mathcal{O}(1-\beta)^{\alpha+1} .
$$

Proposition 6. There are polynomials $P \in S(6, \beta)$ with

$$
|P|_{\beta}=1-(11 / 30)(1-\beta)+(29 / 450)(1-\beta)^{2}+\mathcal{O}(1-\beta)^{5 / 2} .
$$

Proposition 7. Assume the notation of Theorem [1, Then there are real polynomials $P \in S(n+1, \beta)$ with

$$
|P|_{\beta}=1+\left(D_{1}+D_{2} / n\right)(1-\beta)+D(1-\beta)^{2}+\mathcal{O}(1-\beta)^{\alpha+1} .
$$

From the definition of $D$ in Theorem 1 and the constants we will compute at the beginning of section 2 we see that for $n=5$ we have $D_{1}+D_{2} / n=-11 / 30$ and $D+\Delta=29 / 450$, so Propositions [5]and 6 together imply that Theorem 1 is true for $n=5$. Note that for $n \neq 5$ we have $\Delta=0$, so Propositions [5] and 7 taken together imply that Theorem 1 is true for $n \neq 5$.

In [8] it was proved that if $n=5$ and if $\beta$ is sufficiently close to 1 , then maximal polynomials in $S(n+1, \beta)$ (those for which $|P|_{\beta}=r_{n+1}(\beta)$ ) must be nonreal. Taken together, Theorem 1 and Proposition 7 provide strong evidence that this is true only for $n=5$ (although it is conceivable that this could fail for higher-order approximations). 


\section{Preliminaries}

We begin by computing some values (that we will subsequently need) for the constants that appear in Theorem 1, obtaining:

$\begin{array}{cccccc}n & u_{1} & u_{2} & D_{1} & D_{2} & D_{1}+D_{2} / n \\ 3 & 0 & -1 & -1 / 4 & -1 / 4 & -1 / 3 \\ 4 & \frac{-1+\sqrt{5}}{4} & \frac{-1-\sqrt{5}}{4} & -1 / 5 & -2 / 5 & -3 / 10 \\ 5 & -1 / 2 & -1 & -1 / 3 & -1 / 6 & -11 / 30 \\ 6 & -0.2225 & -0.9010 & -0.3014 & & \\ 7 & 0 & -0.7071 & -0.2929 & -0.2929 & -0.3347 \\ 9 & -0.3090 & -0.8090 & & & \\ 10 & -0.1423 & -0.6549 & -0.3138 & & \end{array}$

We next establish some relationships between these constants with

Lemma 8. Assume the notation of Theorem 1. Then

1. $u_{2}<-1 / 2 \leq u_{1}$, and $u_{1} \leq 0$ for $n \neq 4$, and $u_{2}>-1$ for $n \neq 3,5$,

2. $u_{1}+u_{2}<0$ and $u_{1} u_{2}>-1$,

3. $2 n u_{1}+n+1 \geq 1$ and $2 n u_{2}+n+1<0$,

4. $D_{1}<0$ and $D_{2}<0$,

5. $\lim _{n \rightarrow \infty} D_{1}+D_{2} / n=-1 / 3$,

6. $-1<D_{1}+D_{2} / n \leq-3 / 10$, with equality only at $n=4$, and

7. $1+\left(1+D_{1}+D_{2}\right)\left(u_{i}-1\right)-D_{2}\left(2 u_{i}^{2}-2\right)=0$ for $i=1$ and $i=2$.

Proof. From the definition of $k$ in Theorem 1, the relationship between $k$ and $n$ depends on the residue of $n$ modulo 3. For increasing values of $n$ in each of the three residue classes, the sequence $k /(n+1)$ increases to (or is equal to) $1 / 3$ and the sequence $(k+1) /(n+1)$ strictly decreases to $1 / 3$, so the values of $u_{1}$ decrease to (or are equal to) $-1 / 2$ and the values of $u_{2}$ strictly increase to $-1 / 2$. Since the values of $u_{1}$ decrease (or remain constant) in each residue class, and since $u_{1} \leq 0$ for $n=3,5$ and 7 , then $u_{1} \leq 0$ for all $n \neq 4$. Since the values of $u_{2}$ strictly increase in each residue class, and since $u_{2}>-1$ for $n=4$ and $u_{2}=-1$ for $n=3$ and $n=5$, then $u_{2}>-1$ for $n \neq 3,5$. This completes the proof of part 1 of the lemma.

For $n=4$, we have $u_{1}+u_{2}=-1 / 2$ and $u_{1} u_{2}=-1 / 4$. For $n \neq 4$ we have from part 1 that $u_{2}<u_{1} \leq 0$, and part 2 of the lemma follows trivially.

Since $u_{1} \geq-1 / 2$, then $2 n u_{1}+n+1 \geq 1$. For $n=3,4$ and 5 we have $(\mathrm{k}+1) /(\mathrm{n}+1) \leq 1 / 2$. Since in each residue class this quotient strictly decreases to $1 / 3$, then for all $n \geq 3$ we have $2 \pi(k+1) /(n+1) \in(2 \pi / 3, \pi]$. Now $\cos x \leq$ $1 / 2-3 x /(2 \pi)$ on this interval, and from the definition of $k$ in Theorem 1 we know that $k \geq(n-1) / 3$, so

$$
u_{2}=\cos \frac{2 \pi(k+1)}{n+1} \leq \frac{1}{2}-\frac{3(k+1)}{n+1} \leq \frac{1}{2}-\frac{n+2}{n+1}<-\frac{n+1}{2 n}
$$

which completes the proof of part 3 of the lemma.

At $n=4$, we have $D_{1}=-1 / 5$ and $D_{2}=-2 / 5$. For $n \neq 4$ we know from part 1 of Lemma 8 that $u_{2}<u_{1} \leq 0$ so from the definitions of $D_{1}$ and $D_{2}$ in Theorem 1 we see that $D_{1}<0$ and $D_{2}<0$. This completes the proof of part 4 of the lemma.

As $n$ tends to infinity, $u_{1}$ and $u_{2}$ tend to $-1 / 2$, so $D_{1}$ tends to $-1 / 3$ and $D_{2}$ is bounded. This completes the proof of part 5 of the lemma. 
By part 2 of Lemma 8 we have $u_{1}+u_{2}<0$ and $u_{1} u_{2}>-1$. Since by part 4 of Lemma 8 we know that $D_{2}<0$, then

$$
D_{1}+D_{2} / n>D_{1}+D_{2}=-\frac{1+u_{1} u_{2}}{1+u_{1} u_{2}-\left(u_{1}+u_{2}\right)}>-1 \text {. }
$$

From part 1 of Lemma 8 we know that $u_{2}<-1 / 2 \leq u_{1}$, so by computing the partial derivatives of $D_{1}$ we see that $\partial D_{1} / \partial u_{1}>0$ and $\partial D_{1} / \partial u_{2} \leq 0$. Since in each residue class $u_{1}$ decreases to $-1 / 2$ and $u_{2}$ increases to $-1 / 2$, then in each residue class $D_{1}$ decreases to $-1 / 3$. At $n=5,6$ and 10 we have $D_{1}<-3 / 10$, and hence $D_{1}+D_{2} / n<D_{1}<-3 / 10$ for all $n \geq 3$ except possibly $n=3,4$ and 7. Checking the values of $D_{1}+D_{2} / n$ (computed at the beginning of section 2) for these exceptional values completes the proof of part 6 of the lemma.

Expressing $D_{1}$ and $D_{2}$ in terms of $u_{1}$ and $u_{2}$ and simplifying the result verifies part 7, and thus completes the proof of Lemma 8

We now estimate the size of the coefficients of $P^{\prime}$ with

Proposition 9. Suppose that $P \in S(n+1, \beta)$ with $P^{\prime}$ monic and $|P|_{\beta} \geq \beta$. Let $P^{\prime}(z)=\prod_{j=1}^{n}\left(z-\zeta_{j}\right)=z^{n}+a_{n-1} z^{n-1}+\cdots+a_{0}$. Then

1. each $\Re\left[\zeta_{j}\right]=\mathcal{O}(1-\beta)$ and each $\Im\left[\zeta_{j}\right]=\mathcal{O}(1-\beta)^{1 / 2}$,

2. each $a_{n-k}=\mathcal{O}(1-\beta)^{k / 2}$,

3. for $k$ odd, each $\Re\left[a_{n-k}\right]=\mathcal{O}(1-\beta)^{(k+1) / 2}$, and

4. for $k$ even, each $\Im\left[a_{n-k}\right]=\mathcal{O}(1-\beta)^{(k+1) / 2}$.

Proof. Parts 1-3 were proved in [8, Proposition 4]. Part 4 is proved similarly to part 3, by noting that each term of $\Im\left[a_{n-k}\right]$ is a product of $k$ of the $\Re\left[\zeta_{j}\right]$ 's and $\Im\left[\zeta_{j}\right]$ 's, and that for $k$ even, each term has at least one $\Re\left[\zeta_{j}\right]$, so from part 1 of Proposition 9 we have that $\Im\left[a_{n-k}\right]=\mathcal{O}(1-\beta)^{(k+1) / 2}$.

To have $P \in S(n+1, \beta)$ requires that the moduli of the roots of $P$ are all at most 1 . We estimate these moduli with

Proposition 10. Assume the notation of Theorem 1. Let $P$ be a polynomial with $P^{\prime}(z)=z^{n}+a_{n-1} z^{n-1}+\cdots+a_{0}$ and $P(\beta)=0$. Let $z \neq \beta$ be a root of $P$, let $\omega$ be the $(n+1)$ th root of 1 that is closest to $z$ and let $R=(1-\beta)+a_{n-1}\left(\omega^{n}-1\right) / n+$ $\cdots+a_{0}(\omega-1)$.

1. For $0<r \leq 1$, if each $a_{k}=\mathcal{O}(1-\beta)^{r}$, then $|z|^{2}=1-2 \Re[R]+\mathcal{O}(1-\beta)^{2 r}$.

2. Suppose that

$$
\begin{aligned}
& a_{n-1}=n\left(1+D_{1}+D_{2}\right)(1-\beta)+\mathcal{O}(1-\beta)^{\alpha}, \\
& a_{n-2}=-(n-1) D_{2}(1-\beta)+\mathcal{O}(1-\beta)^{\alpha}, \text { and } \\
& a_{n-k}=\mathcal{O}(1-\beta)^{\alpha} \quad \text { for } k \geq 3
\end{aligned}
$$

and define

$$
\begin{gathered}
\Gamma_{2}=2\left(1+D_{1}+D_{2}\right)\left(D_{1}-2 D_{2}+n D_{2}\right) \text { and } \\
\Gamma_{1}=-\Gamma_{2}+\left(-2-4 D_{1}\right) n+\left(1+4 D_{1}-4 D_{2}\right) . \\
\text { If } \Re[\omega]=u_{i} \text { for } i=1 \text { or } i=2 \text {, then } \\
|z|^{2 n+2}=1-2(n+1) \Re[R]+(n+1)\left(\Gamma_{1}+\Gamma_{2} u_{i}\right)(1-\beta)^{2}+\mathcal{O}(1-\beta)^{\alpha+1} .
\end{gathered}
$$


Proof. Since $\beta=1-(1-\beta)$, then by the binomial theorem $\beta^{k}=1-k(1-\beta)+$ $\mathcal{O}(1-\beta)^{2}$. Since $z$ is a root of $P$ we have

$$
0=P(z)=\int_{\beta}^{z} P^{\prime}(t) d t=\frac{z^{n+1}-\beta^{n+1}}{n+1}+a_{n-1} \frac{z^{n}-\beta^{n}}{n}+\cdots+a_{0}(z-\beta),
$$

and solving for $z^{n+1}$ gives us

$$
z^{n+1}=\beta^{n+1}-(n+1)\left[a_{n-1} \frac{z^{n}-\beta^{n}}{n}+\cdots+a_{0}(z-\beta)\right] .
$$

By hypothesis, as $\beta$ goes to 1 the $a_{k}$ all tend to 0 so the roots of $P$ tend to the roots of $z^{n+1}-1$, and so the $\omega$ appearing in the hypotheses is well defined.

Now each $\beta^{k}=1+\mathcal{O}(1-\beta)$, and by the hypothesis of part 1 each $a_{k}=$ $\mathcal{O}(1-\beta)^{r}$. Putting these estimates into equation (2.1), we see that $z^{n+1}=1+$ $\mathcal{O}(1-\beta)^{r}$. Then $z=\omega+\mathcal{O}(1-\beta)^{r}$ and so $\left(z^{k}-\beta^{k}\right) / k=\left(\omega^{k}-1\right) / k+\mathcal{O}(1-\beta)^{r}$. Now note that each $a_{n-k}=\mathcal{O}(1-\beta)^{r}$ and that each $\beta^{k}=1-k(1-\beta)+\mathcal{O}(1-\beta)^{2}$. Substituting these estimates into equation (2.1) gives

$$
\begin{aligned}
z^{n+1} & =1-(n+1)(1-\beta)-(n+1)\left[a_{n-1} \frac{\omega^{n}-1}{n}+\cdots+a_{0}(\omega-1)\right]+\mathcal{O}(1-\beta)^{2 r} \\
& =1-(n+1) R+\mathcal{O}(1-\beta)^{2 r} .
\end{aligned}
$$

Note that $R=\mathcal{O}(1-\beta)^{r}$ so

$$
(1-R)^{n+1}=1-(n+1) R+\mathcal{O}(1-\beta)^{2 r}=z^{n+1}+\mathcal{O}(1-\beta)^{2 r},
$$

so $z=\omega(1-R)+\mathcal{O}(1-\beta)^{2 r}$ and hence $|z|^{2}=z \bar{z}=1-2 \Re[R]+\mathcal{O}(1-\beta)^{2 r}$. This finishes the proof of part 1 .

From the hypotheses of part 2 , we know that $\Re[\omega]=u_{i}$ for $i=1$ or $i=2$. Suppose for the moment that $\Re[\omega]=u_{1}$ and write $\omega=u_{1}+i v_{1}$. Since $\omega^{n+1}=1$, then $|\omega|=1$, so $\omega^{n}=\bar{\omega}$ and $\Re\left[\omega^{2}\right]=2 u_{1}^{2}-1$. Let $A=\left[-\left(1+D_{1}+D_{2}\right)+2 D_{2} u_{1}\right] v_{1}$. From part 7 of Lemma 8 we see that

$$
\Re\left[1+\left(1+D_{1}+D_{2}\right)(\bar{\omega}-1)-D_{2}\left(\bar{\omega}^{2}-1\right)\right]=0
$$

and so using the estimates of the $a_{n-k}$ 's given in the hypotheses of part 2, we get

$$
\begin{aligned}
R & =(1-\beta)+a_{n-1} \frac{\bar{\omega}-1}{n}+a_{n-2} \frac{\bar{\omega}^{2}-1}{n-1}+\cdots+a_{0}(\omega-1) \\
& =\left[1+\left(1+D_{1}+D_{2}\right)(\bar{\omega}-1)-D_{2}\left(\bar{\omega}^{2}-1\right)\right](1-\beta)+\mathcal{O}(1-\beta)^{\alpha} \\
& =i A(1-\beta)+\mathcal{O}(1-\beta)^{\alpha} .
\end{aligned}
$$

The hypotheses of part 2 imply that each $a_{k}=\mathcal{O}(1-\beta)$, so from the proof of part 1 with $r=1$ we have $z=\omega(1-R)+\mathcal{O}(1-\beta)^{2}=\omega[1-i A(1-\beta)]+\mathcal{O}(1-\beta)^{\alpha}$ and so

$$
\left(z^{k}-\beta^{k}\right) / k=\left(\omega^{k}-1\right) / k+\left(1-i A \omega^{k}\right)(1-\beta)+\mathcal{O}(1-\beta)^{\alpha} .
$$

Let $G=n / 2-n\left(1+D_{1}+D_{2}\right)(1-i A \bar{\omega})+(n-1) D_{2}\left(1-i A \bar{\omega}^{2}\right)$. Then from equation (2.1) and the estimates of the $a_{k}$ 's given in the hypotheses of part 2 we 
get

$$
\begin{gathered}
z^{n+1}=1-(n+1)(1-\beta)+\frac{(n+1) n}{2}(1-\beta)^{2} \\
-(n+1)\left[a_{n-1}\left(\frac{\omega^{n}-1}{n}+\left(1-i A \omega^{n}\right)(1-\beta)\right)\right. \\
\quad+a_{n-2}\left(\frac{\omega^{n-1}-1}{n-1}+\left(1-i A \omega^{n-1}\right)(1-\beta)\right) \\
\left.\quad+a_{n-3} \frac{\omega^{n-2}-1}{n-2}+\cdots+a_{0}(\omega-1)\right]+\mathcal{O}(1-\beta)^{\alpha+1} \\
=1-(n+1) R+(n+1) G(1-\beta)^{2}+\mathcal{O}(1-\beta)^{\alpha+1} .
\end{gathered}
$$

Then since $R=i A(1-\beta)+\mathcal{O}(1-\beta)^{\alpha}$ we have

$$
\begin{aligned}
|z|^{2 n+2} & =z^{n+1} \bar{z}^{n+1} \\
& =1-2(n+1) \Re[R]+(n+1)\left[2 \Re[G]+(n+1) A^{2}\right](1-\beta)^{2}+\mathcal{O}(1-\beta)^{\alpha+1} .
\end{aligned}
$$

Thus to complete the proof of part 2 of Proposition 10 for the case $\Re[\omega]=u_{1}$ we need only verify that $2 \Re[G]+(n+1) A^{2}=\Gamma_{1}+\Gamma_{2} u_{1}$.

Let $D_{0}=1+D_{1}+D_{2}$, so from the definition of $A$ we see that

$$
A=\left(-D_{0}+2 D_{2} u_{1}\right) v_{1} .
$$

Note that $\Re[i \bar{\omega}]=\Im[\omega]$. Then from the definition of $G$ we have

$$
\begin{aligned}
\Re[G] & =n / 2-n D_{0}\left(1-A v_{1}\right)+(n-1) D_{2}\left(1-2 A u_{1} v_{1}\right) \\
& =n / 2-n D_{0}+(n-1) D_{2}-A\left[n\left(-D_{0} v_{1}+2 D_{2} u_{1} v_{1}\right)-2 D_{2} u_{1} v_{1}\right] \\
& =\left(-n / 2-n D_{1}-D_{2}\right)-n A^{2}+2 A D_{2} u_{1} v_{1}
\end{aligned}
$$

so

$$
2 \Re[G]+(n+1) A^{2}=\left(-n-2 n D_{1}-2 D_{2}\right)+(-n+1) A^{2}+4 A D_{2} u_{1} v_{1} .
$$

Now

$$
2 D_{2} u_{1}^{2}=\frac{-u_{1}^{2}}{\left(1-u_{1}\right)\left(1-u_{2}\right)}=D_{0} u_{1}+\left(D_{2}-D_{1}\right),
$$

so

$$
\begin{aligned}
A v_{1} & =\left(-D_{0}+2 D_{2} u_{1}\right)\left(1-u_{1}^{2}\right) \\
& =-D_{0}+2 D_{2} u_{1}-u_{1}\left(-D_{0} u_{1}+2 D_{2} u_{1}^{2}\right) \\
& =-D_{0}+\left(D_{1}+D_{2}\right) u_{1} .
\end{aligned}
$$

Using these two equalities, we see that

$$
\begin{aligned}
A^{2} & =\left(-D_{0}+2 D_{2} u_{1}\right)\left[-D_{0}+\left(D_{1}+D_{2}\right) u_{1}\right] \\
& =D_{0}^{2}+\left(-D_{0} D_{1}-3 D_{0} D_{2}\right) u_{1}+\left(D_{1}+D_{2}\right)\left(2 D_{2} u_{1}^{2}\right) \\
& =D_{0}^{2}-D_{1}^{2}+D_{2}^{2}-2 D_{0} D_{2} u_{1}
\end{aligned}
$$

and

$$
\begin{aligned}
2 A D_{2} u_{1} v_{1} & =2 D_{2} u_{1}\left[-D_{0}+\left(D_{1}+D_{2}\right) u_{1}\right] \\
& =-2 D_{0} D_{2} u_{1}+\left(D_{1}+D_{2}\right)\left[D_{0} u_{1}+\left(D_{2}-D_{1}\right)\right] \\
& =D_{0}\left(D_{1}-D_{2}\right) u_{1}+\left(D_{2}^{2}-D_{1}^{2}\right) .
\end{aligned}
$$


Thus from equation (2.2) we have

$$
\begin{aligned}
2 \Re[G]+(n+1) A^{2}= & \left(-n-2 n D_{1}-2 D_{2}\right)+(-n+1)\left(D_{0}^{2}-D_{1}^{2}+D_{2}^{2}-2 D_{0} D_{2} u_{1}\right) \\
& \quad+2\left[D_{0}\left(D_{1}-D_{2}\right) u_{1}+\left(D_{2}^{2}-D_{1}^{2}\right)\right] \\
= & \left(-1-2 D_{1}-D_{0}^{2}+D_{1}^{2}-D_{2}^{2}\right) n+\left(-2 D_{2}+D_{0}^{2}-3 D_{1}^{2}+3 D_{2}^{2}\right) \\
& \quad+2 D_{0} u_{1}\left(D_{1}-2 D_{2}+n D_{2}\right) \\
= & \Gamma_{1}+\Gamma_{2} u_{1} .
\end{aligned}
$$

This finishes the proof of part 2 of Proposition 10 for the case $\Re[\omega]=u_{1}$. Since $D_{1}$ and $D_{2}$ are symmetric in $u_{1}$ and $u_{2}$, swapping $u_{1}$ and $u_{2}$ in this proof verifies part 2 of Proposition [10] for the remaining case $\Re[\omega]=u_{2}$, and thus completes the proof of Proposition 10

Finally, consider the linear transformation $\mathcal{T}$ which takes functions to real numbers via

$$
\mathcal{T}(f)=\frac{\left(2 n u_{1}+n+1\right) f\left(u_{2}\right)-\left(2 n u_{2}+n+1\right) f\left(u_{1}\right)}{2\left(u_{1}-u_{2}\right)} .
$$

Recall that by Lemma 8 we have $u_{1}-u_{2}>0,2 n u_{1}+n+1>0$ and $2 n u_{2}+n+1<0$, so $\mathcal{T} / n$ is a weighted average. This implies that $\mathcal{T}$ preserves inequalities, in the sense that if $f\left(u_{1}\right) \leq g\left(u_{1}\right)$ and $f\left(u_{2}\right) \leq g\left(u_{2}\right)$, then $\mathcal{T}(f) \leq \mathcal{T}(g)$.

In the process of analyzing several inequalities, we will need the following values of the transformation $\mathcal{T}$ :

$$
\begin{aligned}
\mathcal{T}(1) & =n, \\
\mathcal{T}(2+2 u) & =n-1, \\
\mathcal{T}\left(1+4 u+4 u^{2}\right) & =-\left[n+2+2(n+1)\left(u_{1}+u_{2}\right)+4 n u_{1} u_{2}\right] \\
& =-\frac{n+1+D_{1}+3 n D_{1}+3 D_{2}}{D_{2}}, \\
\mathcal{T}\left(\frac{1}{1-u}\right) & =n+n D_{1}+D_{2}, \\
\mathcal{T}\left(\frac{u}{1-u}\right) & =n D_{1}+D_{2} .
\end{aligned}
$$

We will also use the results of

Lemma 11. For the linear transformation $\mathcal{T}$ defined in equation (2.3) we have

1. $\mathcal{T}\left(1+4 u+4 u^{2}\right) /(n-2)<1 / 2$ for $n \neq 3,4$ and 6 , and

2. $\mathcal{T}\left(8 u^{2}+8 u^{3}\right) \geq 0$ for all $n$.

Proof. From the formula for $\mathcal{T}\left(1+4 u+4 u^{2}\right)$ in (2.4) and from part 3 of Lemma 8 we have

$$
\begin{aligned}
& \partial \mathcal{T}\left(1+4 u+4 u^{2}\right) / \partial u_{1}=-2\left(2 n u_{2}+n+1\right)>0 \text { and } \\
& \partial \mathcal{T}\left(1+4 u+4 u^{2}\right) / \partial u_{2}=-2\left(2 n u_{1}+n+1\right)<0 .
\end{aligned}
$$

Recall from the proof of Lemma 8 that for each residue class of $n$ modulo 3 the values of $u_{1}$ decrease and the values of $u_{2}$ increase, so the signs of the partial derivatives above imply that in each residue class the values of $\mathcal{T}\left(1+4 u+4 u^{2}\right)$ decrease. Since $1+4 u+4 u^{2}=(1+2 u)^{2} \geq 0$ and since $\mathcal{T}$ preserves inequalities, then $\mathcal{T}\left(1+4 u+4 u^{2}\right) \geq 0$, so the values of $\mathcal{T}\left(1+4 u+4 u^{2}\right) /(n-2)$ also decrease 
in each residue class. Using the formula for $\mathcal{T}\left(1+4 u+4 u^{2}\right)$ in (2.4) and the values of the $u_{i}$ computed at the beginning of section 2 , we calculate the values of $\mathcal{T}\left(1+4 u+4 u^{2}\right) /(n-2)$ at $n=5,7$ and 9 , getting respectively $1 / 3,0.4627$ and 0.3372 . Since they are all less than $1 / 2$, this proves part 1 of Lemma 11 ,

Since by definition $u_{i} \geq-1$, then $8 u_{i}^{2}+8 u_{i}^{3}=8 u_{i}^{2}\left(1+u_{i}\right) \geq 0$ for both $i=1$ and $i=2$, and so part 2 of Lemma 11 follows from our observation that $\mathcal{T}$ preserves inequalities.

Finally, we will deal with polynomials that are "almost" in $S(n, \beta)$ using

Lemma 12. Suppose that $P$ is a polynomial of degree $n$ with all roots in $\{z:|z| \leq$ $\left.1+\mathcal{O}(1-\beta)^{r}\right\}$, one root at $\beta$, and all other roots bounded away from $\beta$. Then there is a polynomial $Q \in S(n, \beta)$ such that $|Q|_{\beta}=|P|_{\beta}+\mathcal{O}(1-\beta)^{r}$.

Proof. If $P \in S(n, \beta)$, then we may take $Q=P$. If not, then at least one root of $P$ has modulus greater than 1 . In this case, let

$$
c=\max \left\{\frac{|z|^{2}-1}{|z-\beta|^{2}}: z \text { is a root of } P \text { and }|z|>1\right\} .
$$

Since by hypothesis $|z-\beta|$ is bounded away from 0 and $|z| \leq 1+\mathcal{O}(1-\beta)^{r}$, then $0<c \leq \mathcal{O}(1-\beta)^{r}$. In particular, for $\beta$ sufficiently close to 1 we have $0<c<1$.

Let $Q$ be the polynomial with roots $\{z-c(z-\beta): z$ is a root of $P\}$. Since the mapping $z \mapsto z-c(z-\beta)$ is a contraction of the plane that leaves $\beta$ fixed and moves all roots of $P$ (and hence $P^{\prime}$ ) toward $\beta$ by at most $\mathcal{O}(1-\beta)^{r}$, then $Q(\beta)=0$ and $|Q|_{\beta}=|P|_{\beta}+\mathcal{O}(1-\beta)^{r}$. Thus we need only show that all roots of $Q$ are in the unit disk.

Note that for $t$ real the image of the mapping $t \mapsto z-t(z-\beta)$ is a line, with $t=0$ mapping to $z$, and $t=1$ mapping to $\beta$, and $t=\left(|z|^{2}-1\right) /|z-\beta|^{2}$ mapping to

$$
z-\frac{|z|^{2}-1}{|z-\beta|^{2}}(z-\beta)=z-\frac{z \bar{z}-1}{\bar{z}-\beta}=\frac{1-\beta z}{\bar{z}-\beta} .
$$

If $z$ is in the unit disk, then the images of every $t$ between 0 and 1 lie on the line between $z$ and $\beta$, hence in the unit disk. If $z$ is not in the unit disk, then $|(1-\beta z) /(z-\beta)|<1$ and so the images of every $t$ between $\left(|z|^{2}-1\right) /|z-\beta|^{2}$ and 1 lie on the line between $(1-\beta z) /(\bar{z}-\beta)$ and $\beta$, hence in the unit disk. Thus for every root $z$ of $P$, the image of $c$ lies in the unit disk, so all roots of $Q$ are in the unit disk and so $Q \in S(n, \beta)$. This completes the proof of Lemma 12.

\section{Proof of Proposition 5}

Take any $P \in S(n+1, \beta)$, assume without loss of generality that $P^{\prime}$ is monic, and write $P^{\prime}(z)=\prod_{j=1}^{n}\left(z-\zeta_{j}\right)=z^{n}+a_{n-1} z^{n-1}+\cdots+a_{0}$.

If $|P|_{\beta} \leq 1+\left(D_{1}+D_{2} / n\right)(1-\beta)+(D+\Delta)(1-\beta)^{2}$, then Proposition 5 is trivially true. Thus we may assume without loss of generality that

$$
|P|_{\beta} \geq 1+\left(D_{1}+D_{2} / n\right)(1-\beta)+(D+\Delta)(1-\beta)^{2} .
$$

From part 6 of Lemma 8 we have that $D_{1}+D_{2} / n>-1$, and so inequality (3.1) implies that $|P|_{\beta} \geq \beta$ as long as $\beta$ is sufficiently close to 1 . Note that $P$ thus satisfies all the hypotheses of Proposition 9 , 
We begin by estimating some relationships between the coefficients of $P^{\prime}$ with

Lemma 13. Suppose that $\Im\left[a_{n-1}\right]=\mathcal{O}(1-\beta)^{3 / 2}$ and that each

$$
\left|\zeta_{j}-\beta\right|=1+\left(D_{1}+D_{2} / n\right)(1-\beta)+\mathcal{O}(1-\beta)^{2} .
$$

Then

1. $\Im\left[a_{n-2}\right]=(-3 / 2) \Im\left[a_{n-3}\right]+\mathcal{O}(1-\beta)^{5 / 2}$ and

2. $\Re\left[a_{n-3}\right]+2 \Re\left[a_{n-4}\right]=(n-2)\left(1+D_{1}+D_{2} / n\right)(1-\beta) \Re\left[a_{n-2}\right]+\mathcal{O}(1-\beta)^{3}$.

Proof. Let each $\zeta_{j}=x_{j}+i y_{j}$ and note that by Proposition 9 we have $x_{j}=\mathcal{O}(1-\beta)$ and $y_{j}=\mathcal{O}(1-\beta)^{1 / 2}$. Note that by hypothesis, $\sum_{i} y_{i}=-\Im\left[a_{n-1}\right]=\mathcal{O}(1-\beta)^{3 / 2}$ and that each

$$
\left(\beta-x_{j}\right)^{2}+y_{j}^{2}=\left|\beta-\zeta_{j}\right|^{2}=1+2\left(D_{1}+D_{2} / n\right)(1-\beta)+\mathcal{O}(1-\beta)^{2},
$$

so solving for $x_{j}$ gives us

$$
x_{j}=y_{j}^{2} / 2-\left(1+D_{1}+D_{2} / n\right)(1-\beta)+\mathcal{O}(1-\beta)^{2} .
$$

Note that $\Im\left[a_{n-3}\right]=-\sum_{i<j<k} \Im\left[\zeta_{i} \zeta_{j} \zeta_{k}\right]=\sum_{i<j<k} y_{i} y_{j} y_{k}+\mathcal{O}(1-\beta)^{5 / 2}$, so

$$
\begin{aligned}
\mathcal{O}(1-\beta)^{5 / 2} & =\sum_{i} y_{i} \sum_{i<j} y_{i} y_{j}=\sum_{i \neq j} y_{i}^{2} y_{j}+3 \sum_{i<j<k} y_{i} y_{j} y_{k} \\
& =\sum_{i \neq j} y_{i}^{2} y_{j}+3 \Im\left[a_{n-3}\right]+\mathcal{O}(1-\beta)^{5 / 2}
\end{aligned}
$$

and so $\sum_{i \neq j} y_{i}^{2} y_{j}=-3 \Im\left[a_{n-3}\right]+\mathcal{O}(1-\beta)^{5 / 2}$. Then using equation (3.2) we have

$$
\begin{aligned}
\Im\left[a_{n-2}\right] & =\sum_{i<j} \Im\left[\zeta_{i} \zeta_{j}\right]=\sum_{i \neq j} x_{i} y_{j} \\
& =(1 / 2) \sum_{i \neq j} y_{i}^{2} y_{j}-\left(1+D_{1}+D_{2} / n\right)(1-\beta) \sum_{i \neq j} y_{j}+\mathcal{O}(1-\beta)^{5 / 2} \\
& =(-3 / 2) \Im\left[a_{n-3}\right]+\mathcal{O}(1-\beta)^{5 / 2},
\end{aligned}
$$

which completes the proof of part 1 of Lemma 13

Let $S$ be the set of triples $(i, j, k)$ of distinct integers from 1 to $n$ with $j<k$. Note that $\Re\left[a_{n-2}\right]=\sum_{i<j} \Re\left[\zeta_{i} \zeta_{j}\right]=-\sum_{i<j} y_{i} y_{j}+\mathcal{O}(1-\beta)^{2}$ and $\Re\left[a_{n-3}\right]=$ $-\sum_{i<j<k} \Re\left[\zeta_{i} \zeta_{j} \zeta_{k}\right]=\sum_{S} x_{i} y_{j} y_{k}+\mathcal{O}(1-\beta)^{3}$. Furthermore,

$$
\mathcal{O}(1-\beta)^{3}=\sum_{i} y_{i} \sum_{j<k<l} y_{j} y_{k} y_{l}=\sum_{S} y_{i}^{2} y_{j} y_{k}+4 \sum_{i<j<k<l} y_{i} y_{j} y_{k} y_{l},
$$

so

$$
\begin{aligned}
\Re\left[a_{n-4}\right] & =\sum_{i<j<k<l} \Re\left[\zeta_{i} \zeta_{j} \zeta_{k} \zeta_{l}\right]=\sum_{i<j<k<l} y_{i} y_{j} y_{k} y_{l}+\mathcal{O}(1-\beta)^{3} \\
& =(-1 / 4) \sum_{S} y_{i}^{2} y_{j} y_{k}+\mathcal{O}(1-\beta)^{3} .
\end{aligned}
$$


Then using equation (3.2) we have

$$
\begin{aligned}
\Re\left[a_{n-3}\right]+2 \Re\left[a_{n-4}\right] & =\sum_{S}\left(x_{i}-y_{i}^{2} / 2\right) y_{j} y_{k}+\mathcal{O}(1-\beta)^{3} \\
& =-\left(1+D_{1}+D_{2} / n\right)(1-\beta)(n-2) \sum_{j<k} y_{j} y_{k}+\mathcal{O}(1-\beta)^{3} \\
& =(n-2)\left(1+D_{1}+D_{2} / n\right)(1-\beta) \Re\left[a_{n-2}\right]+\mathcal{O}(1-\beta)^{3},
\end{aligned}
$$

which completes the proof of Lemma13.

We now establish a lower bound on $\Re\left[a_{n-4}\right]$ with

Lemma 14. Suppose that

$$
\begin{aligned}
& \Im\left[a_{n-1}\right]=\mathcal{O}(1-\beta)^{\alpha}, \\
& \Re\left[a_{n-2}\right]=-(n-1) D_{2}(1-\beta)+\mathcal{O}(1-\beta)^{\alpha}, \text { and } \\
& \Im\left[a_{n-3}\right]=\mathcal{O}(1-\beta)^{\alpha} .
\end{aligned}
$$

If $n=5$, then define $\delta=-1 / 15$; otherwise define $\delta=0$. Then

$$
\Re\left[a_{n-4}\right] \geq \delta(1-\beta)^{2}+\mathcal{O}(1-\beta)^{\alpha+1} .
$$

Proof. Let each $\zeta_{j}=x_{j}+i y_{j}$ and recall by Proposition 9 that $x_{j}=\mathcal{O}(1-\beta)$ and $y_{j}=\mathcal{O}(1-\beta)^{1 / 2}$. Let $F(y)=\prod_{i=1}^{n}\left(y+y_{i}\right)=y^{n}+b_{n-1} y^{n-1}+\cdots+b_{0}$. Note that

$$
\begin{aligned}
\Re\left[a_{n-4}\right] & =\sum_{i<j<k<l} \Re\left[\zeta_{i} \zeta_{j} \zeta_{k} \zeta_{l}\right]=\sum_{i<j<k<l} y_{i} y_{j} y_{k} y_{l}+\mathcal{O}(1-\beta)^{3} \\
& =b_{n-4}+\mathcal{O}(1-\beta)^{3}
\end{aligned}
$$

and that by hypothesis

$$
\begin{aligned}
b_{n-1} & =\sum_{i} y_{i}=\sum_{i} \Im\left[\zeta_{i}\right]=-\Im\left[a_{n-1}\right] \\
& =\mathcal{O}(1-\beta)^{\alpha}, \\
b_{n-2} & =\sum_{i<j} y_{i} y_{j}=-\sum_{i<j} \Re\left[\zeta_{i} \zeta_{j}\right]+\mathcal{O}(1-\beta)^{2}=-\Re\left[a_{n-2}\right]+\mathcal{O}(1-\beta)^{2} \\
& =(n-1) D_{2}(1-\beta)+\mathcal{O}(1-\beta)^{\alpha}, \text { and } \\
b_{n-3} & =\sum_{i<j<k} y_{i} y_{j} y_{k}=-\sum_{i<j<k} \Im\left[\zeta_{i} \zeta_{j} \zeta_{k}\right]+\mathcal{O}(1-\beta)^{5 / 2}=\Im\left[a_{n-3}\right]+\mathcal{O}(1-\beta)^{5 / 2} \\
& =\mathcal{O}(1-\beta)^{\alpha} .
\end{aligned}
$$

Let

$$
\begin{aligned}
f(y) & =F^{(n-4)}(y) \\
& =\frac{n !}{24} y^{4}+\frac{(n-1) !}{6} b_{n-1} y^{3}+\frac{(n-2) !}{2} b_{n-2} y^{2}+(n-3) ! b_{n-3} y+(n-4) ! b_{n-4} .
\end{aligned}
$$

Now by definition $F$ has all real roots, hence by Rolle's Theorem (from elementary calculus) so does $f$. Then the "reverse" of $f$ defined by $y^{4} f(1 / y)=$ $(n-4) ! b_{n-4} y^{4}+\cdots+n ! / 24$ has all real roots, so by Rolle's theorem so does the reverse's second derivative

$$
12(n-4) ! b_{n-4} y^{2}+6(n-3) ! b_{n-3} y+(n-2) ! b_{n-2} .
$$


Since this quadratic has all real roots, then its discriminant is nonnegative, so

$$
\left[6(n-3) ! b_{n-3}\right]^{2}-48(n-2) !(n-4) ! b_{n-2} b_{n-4} \geq 0 .
$$

Using our estimates of the $b_{n-k}$ 's (including $b_{n-4}=\mathcal{O}(1-\beta)^{2}$ ), this implies that $-D_{2}(1-\beta) b_{n-4} \geq \mathcal{O}(1-\beta)^{2 \alpha}$ and so $b_{n-4} \geq \mathcal{O}(1-\beta)^{2 \alpha-1}$. Now for $n \neq 3,5$ we have $\alpha=2$ and so $\Re\left[a_{n-4}\right]=b_{n-4}+\mathcal{O}(1-\beta)^{3} \geq \mathcal{O}(1-\beta)^{3}$, which finishes the proof of Lemma 14 for these values of $n$.

Lemma 14 is trivially true for $n=3$, since then $\Re\left[a_{n-4}\right] \equiv 0 \geq \mathcal{O}(1-\beta)^{5 / 2}$.

Finally, for $n=5$ we have that

$$
f(y)=5 y^{4}+4 b_{n-1} y^{3}+3 b_{n-2} y^{2}+2 b_{n-3} y+b_{n-4}
$$

has all real roots, hence by Rolle's theorem so does its derivative

$$
f^{\prime}(y)=20 y^{3}+12 b_{n-1} y^{2}+6 b_{n-2} y+2 b_{n-3} .
$$

A classical result (see e.g. [11, p. 289]) states that if a cubic polynomial $a x^{3}+$ $b x^{2}+c x+d$ has all real roots, then its discriminant is nonnegative, so

$$
18 a b c d-4 b^{3} d+b^{2} c^{2}-4 a c^{3}-27 a^{2} d^{2} \geq 0 .
$$

Applying this to $f^{\prime}(y)$, we have

$$
-4[20]\left[6 b_{n-2}\right]^{3}-27[20]^{2}\left[2 b_{n-3}\right]^{2} \geq \mathcal{O}(1-\beta)^{4},
$$

which implies that $2 b_{n-2}^{3}+5 b_{n-3}^{2} \leq \mathcal{O}(1-\beta)^{4}$. Since for $n=5$ we have $D_{2}=-1 / 6$, then by hypothesis $b_{n-2}=(-2 / 3)(1-\beta)+\mathcal{O}(1-\beta)^{3 / 2}$, and so

$$
\begin{aligned}
b_{n-3}^{2} & \leq(-2 / 5) b_{n-2}^{3}+\mathcal{O}(1-\beta)^{4} \\
& =(16 / 135)(1-\beta)^{3}+\mathcal{O}(1-\beta)^{7 / 2} .
\end{aligned}
$$

We also have that the first derivative of the reverse of $f$

$$
4 b_{n-4} y^{3}+6 b_{n-3} y^{2}+6 b_{n-2} y+4 b_{n-1}
$$

has all real roots, so applying our classical result gives

$$
\left[6 b_{n-3}\right]^{2}\left[6 b_{n-2}\right]^{2}-4\left[4 b_{n-4}\right]\left[6 b_{n-2}\right]^{3} \geq \mathcal{O}(1-\beta)^{6} .
$$

Dividing this by $144 b_{n-2}^{2}$ and recalling that $b_{n-2}=(-2 / 3)(1-\beta)+\mathcal{O}(1-\beta)^{3 / 2}$ yields

$$
9 b_{n-3}^{2}+16(1-\beta) b_{n-4} \geq \mathcal{O}(1-\beta)^{7 / 2} .
$$

Combining these two inequalities implies that for $n=5$ we have

$$
\begin{aligned}
\Re\left[a_{n-4}\right] & =b_{n-4}+\mathcal{O}(1-\beta)^{3} \\
& \geq \frac{-9 b_{n-3}^{2}}{16(1-\beta)}+\mathcal{O}(1-\beta)^{5 / 2} \\
& \geq(-1 / 15)(1-\beta)^{2}+\mathcal{O}(1-\beta)^{5 / 2} .
\end{aligned}
$$

This completes the proof of Lemma 14 .

We now begin the proof of Proposition [5. Our first step will be to show that $|P|_{\beta} \leq 1+\left(D_{1}+D_{2} / n\right)(1-\beta)+\mathcal{O}(1-\beta)^{2}$. Recall that $P$ satisfies the hypotheses of Proposition 9, so each $a_{n-k}=\mathcal{O}(1-\beta)^{k / 2}$. Let $\omega \neq 1$ be any $(n+1)$ st root of 1 and let $z$ be the root of $P($ so $|z| \leq 1)$ closest to $\omega$. Then in Proposition 10 we have

$$
\begin{aligned}
R & =(1-\beta)+a_{n-1}\left(\omega^{n}-1\right) / n+\cdots+a_{0}(\omega-1) \\
& =a_{n-1}\left(\omega^{n}-1\right) / n+\mathcal{O}(1-\beta)
\end{aligned}
$$


and so by part 1 of Proposition 10 with $r=1 / 2$, we have

$$
|z|^{2}=1-2 \Re\left[a_{n-1}\left(\omega^{n}-1\right) / n\right]+\mathcal{O}(1-\beta) .
$$

Since $|z| \leq 1$ and $\omega^{n}=\bar{\omega}$, this implies that $\Re\left[a_{n-1}(\bar{\omega}-1)\right] \geq \mathcal{O}(1-\beta)$. Expanding the product and noting that by Proposition 9 we have $\Re\left[a_{n-1}\right]=\mathcal{O}(1-\beta)$, we get that $\Im\left[a_{n-1}\right] \Im[\omega] \geq \mathcal{O}(1-\beta)$. Choosing $\omega$ non-real and repeating this argument with $\bar{\omega}$ substituted for $\omega$ provides that $\Im\left[a_{n-1}\right] \Im[\bar{\omega}] \geq \mathcal{O}(1-\beta)$ and so $\Im\left[a_{n-1}\right]=\mathcal{O}(1-\beta)$. Thus we have $a_{n-1}=\mathcal{O}(1-\beta)$.

Recall that each $a_{n-k}=\mathcal{O}(1-\beta)^{k / 2}$, so we know now that each $a_{n-k}=$ $\mathcal{O}(1-\beta)$. Since $\omega^{n-k}=\bar{\omega}^{k+1}$, by part 1 of Proposition 10 with $r=1$ we have

$$
|z|^{2}=1-2 \Re\left[(1-\beta)+a_{n-1} \frac{\bar{\omega}-1}{n}+a_{n-2} \frac{\bar{\omega}^{2}-1}{n-1}+a_{n-3} \frac{\bar{\omega}^{3}-1}{n-2}\right]+\mathcal{O}(1-\beta)^{2} .
$$

Since $|z| \leq 1$ this implies that

$$
-\Re\left[a_{n-1} \frac{\bar{\omega}-1}{n}+a_{n-2} \frac{\bar{\omega}^{2}-1}{n-1}+a_{n-3} \frac{\bar{\omega}^{3}-1}{n-2}\right] \leq(1-\beta)+\mathcal{O}(1-\beta)^{2} .
$$

Averaging the expressions obtained by substituting $\omega$ and $\omega$ into inequality (3.3) and noting that by Proposition 9 we have $\Re\left[a_{n-3}\right]=\mathcal{O}(1-\beta)^{2}$ we get

$$
\Re\left[a_{n-1}\right] \Re\left[\frac{1-\omega}{n}\right]+\Re\left[a_{n-2}\right] \Re\left[\frac{1-\omega^{2}}{n-1}\right] \leq(1-\beta)+\mathcal{O}(1-\beta)^{2} .
$$

Let $u=\Re[\omega]$. Note that since $|\omega|=1$, then $\Re\left[\omega^{2}\right]=2 u^{2}-1$, so dividing inequality (3.4) by $1-u$, we get

$$
\frac{\Re\left[a_{n-1}\right]}{n}+\frac{\Re\left[a_{n-2}\right]}{n-1}(2+2 u) \leq \frac{1-\beta}{1-u}+\mathcal{O}(1-\beta)^{2}
$$

for each $\omega \neq 1$. In particular, inequality (3.5) holds for $u=u_{1}$ and $u=u_{2}$ as defined in Theorem 1

Applying the linear transformation $\mathcal{T}$ defined in equation (2.3) to inequality (3.5), and using the values computed in (2.4), we see that

$$
\Re\left[a_{n-1}\right]+\Re\left[a_{n-2}\right] \leq\left(n+n D_{1}+D_{2}\right)(1-\beta)+\mathcal{O}(1-\beta)^{2} .
$$

Recall that $P^{\prime}(z)=\prod_{j=1}^{n}\left(z-\zeta_{j}\right)=z^{n}+a_{n-1} z^{n-1}+\cdots+a_{0}$, that each $a_{n-k}=$ $\mathcal{O}(1-\beta)$ and that $\Re\left[a_{n-3}\right]=\mathcal{O}(1-\beta)^{2}$. Then

$$
\begin{aligned}
|P|_{\beta}^{2 n} & =\left(\min _{j}\left|\beta-\zeta_{j}\right|\right)^{2 n} \leq \prod_{j=1}^{n}\left|\beta-\zeta_{j}\right|^{2}=\left|P^{\prime}(\beta)\right|^{2} \\
& =P^{\prime}(\beta) \bar{P}^{\prime}(\beta)=\beta^{2 n}+2 \Re\left[a_{n-1}\right] \beta^{2 n-1}+2 \Re\left[a_{n-2}\right] \beta^{2 n-2}+\mathcal{O}(1-\beta)^{2} \\
& =1-2 n(1-\beta)+2 \Re\left[a_{n-1}\right]+2 \Re\left[a_{n-2}\right]+\mathcal{O}(1-\beta)^{2} \\
& =\left[1-(1-\beta)+\left(\Re\left[a_{n-1}\right]+\Re\left[a_{n-2}\right]\right) / n\right]^{2 n}+\mathcal{O}(1-\beta)^{2}
\end{aligned}
$$

and so using inequalities (3.7) and then (3.6) we have

$$
\begin{aligned}
|P|_{\beta} & \leq 1-(1-\beta)+\left(\Re\left[a_{n-1}\right]+\Re\left[a_{n-2}\right]\right) / n+\mathcal{O}(1-\beta)^{2} \\
& \leq 1+\left(D_{1}+D_{2} / n\right)(1-\beta)+\mathcal{O}(1-\beta)^{2} .
\end{aligned}
$$

This completes our first step. 
Our second step will be to verify the hypotheses of part 2 of Proposition 10] by showing that

$$
\begin{aligned}
& a_{n-1}=n\left(1+D_{1}+D_{2}\right)(1-\beta)+\mathcal{O}(1-\beta)^{\alpha}, \\
& a_{n-2}=-(n-1) D_{2}(1-\beta)+\mathcal{O}(1-\beta)^{\alpha}, \text { and } \\
& a_{n-k}=\mathcal{O}(1-\beta)^{\alpha} \text { for } k \geq 3 .
\end{aligned}
$$

Combining inequalities (3.1) and (3.8), we see that

$$
|P|_{\beta}=1+\left(D_{1}+D_{2} / n\right)(1-\beta)+\mathcal{O}(1-\beta)^{2} .
$$

Since equation (3.8) is thus an equality, then so are equations (3.7) and (3.6), and thus equation (3.5) for $u=u_{i}$ and equations (3.4) and (3.3) for $\Re[\omega]=u_{i}$.

Since equation (3.5) is an equality for $u=u_{i}$, we can solve the resulting linear system in the variables $\Re\left[a_{n-1}\right]$ and $\Re\left[a_{n-2}\right]$ and get

$$
\begin{aligned}
\Re\left[a_{n-1}\right] & =\frac{-n\left(u_{1}+u_{2}\right)}{\left(1-u_{1}\right)\left(1-u_{2}\right)}(1-\beta)+\mathcal{O}(1-\beta)^{2} \\
& =n\left(1+D_{1}+D_{2}\right)(1-\beta)+\mathcal{O}(1-\beta)^{2} \text { and } \\
\Re\left[a_{n-2}\right] & =\frac{n-1}{2\left(1-u_{1}\right)\left(1-u_{2}\right)}(1-\beta)+\mathcal{O}(1-\beta)^{2} \\
& =-(n-1) D_{2}(1-\beta)+\mathcal{O}(1-\beta)^{2} .
\end{aligned}
$$

Note that from Proposition 9 we have that $\Re\left[a_{n-k}\right]=\mathcal{O}(1-\beta)^{2}$ for $k \geq 3$, so we now have the correct real parts for our second step. Thus we need only show that each $\Im\left[a_{n-k}\right]=\mathcal{O}(1-\beta)^{\alpha}$.

Recalling the definitions of $u_{1}$ and $u_{2}$ in Theorem 11 we can choose $\omega_{1}$ and $\omega_{2}$ to be $(n+1)$ st roots of 1 so that $\Re\left[\omega_{i}\right]=u_{i}$. For $\omega=\omega_{i}$, expanding the products in equality (3.3) and cancelling those terms of equality (3.4) gives us

$$
\frac{\Im\left[a_{n-1}\right]}{n} \Im\left[\omega_{i}\right]+\frac{\Im\left[a_{n-2}\right]}{n-1} \Im\left[\omega_{i}^{2}\right]+\frac{\Im\left[a_{n-3}\right]}{n-2} \Im\left[\omega_{i}^{3}\right]=\mathcal{O}(1-\beta)^{2} .
$$

Consider the case $i=1$. Since $\left|\omega_{1}\right|=1$ and since by part 1 of Lemma 8 we have $-1 / 2 \leq u_{1}<1$, then $\Im\left[\omega_{1}\right] \neq 0$. Now by Proposition $9 \Im\left[a_{n-k}\right]=\mathcal{O}(1-\beta)^{3 / 2}$ for $k \geq 2$, so equation (3.10) implies that $\Im\left[a_{n-1}\right]=\mathcal{O}(1-\beta)^{3 / 2}$. If $n=3$ or $n=5$, then by definition $\alpha=3 / 2$, so this completes our second step for those two values of $n$.

Assume then without loss of generality that $n \neq 3,5$. Again by part 1 of Lemma 8 we have $-1<u_{2}<u_{1}<1$ so $\Im\left[\omega_{i}\right] \neq 0$. Thus we may divide equation (3.10) by $\Im\left[\omega_{i}\right]$ to obtain

$$
\frac{\Im\left[a_{n-1}\right]}{n}+\frac{\Im\left[a_{n-2}\right]}{n-1}\left(2 u_{i}\right)+\frac{\Im\left[a_{n-3}\right]}{n-2}\left(4 u_{i}^{2}-1\right)=\mathcal{O}(1-\beta)^{2} .
$$

Now subtracting equality (3.11) with $i=2$ from equality (3.11) with $i=1$ and dividing by $2\left(u_{1}-u_{2}\right)$ produces

$$
\frac{\Im\left[a_{n-2}\right]}{n-1}+\frac{\Im\left[a_{n-3}\right]}{n-2} 2\left(u_{1}+u_{2}\right)=\mathcal{O}(1-\beta)^{2} .
$$

Since equation (3.7) is an equality, we have each $\left|\beta-\zeta_{j}\right|=|P|_{\beta}+\mathcal{O}(1-\beta)^{2}$. Recall that $\Im\left[a_{n-1}\right]=\mathcal{O}(1-\beta)^{3 / 2}$ and that $|P|_{\beta}=1+\left(D_{1}+D_{2} / n\right)(1-\beta)+$ 
$\mathcal{O}(1-\beta)^{2}$. Then by part 1 of Lemma 13$]$ we have $\Im\left[a_{n-2}\right]=(-3 / 2) \Im\left[a_{n-3}\right]+$ $\mathcal{O}(1-\beta)^{5 / 2}$, so substituting into (3.12) we have

$$
\Im\left[a_{n-3}\right]\left[\frac{-3 / 2}{n-1}+\frac{2\left(u_{1}+u_{2}\right)}{n-2}\right]=\mathcal{O}(1-\beta)^{2} .
$$

Now by part 2 of Lemma 8 we have $u_{1}+u_{2}<0$ so the quantity in brackets is non-zero. Then $\Im\left[a_{n-3}\right]=\mathcal{O}(1-\beta)^{2}$, and so solving back in equations (3.12) and (3.11) we find that $\Im\left[a_{n-k}\right]=\mathcal{O}(1-\beta)^{2}$ for all $k \leq 3$. Note that by Proposition 9 we have $a_{n-k}=\mathcal{O}(1-\beta)^{2}$ for all $k \geq 4$, and so $\Im\left[a_{n-k}\right]=\mathcal{O}(1-\beta)^{2}$ for all $k$. Since $n \neq 3,5$, then by definition $\alpha=2$, and so this finishes the proof of our second step.

We will now finish the proof of Proposition 5 Consider only those roots $z$ of $P$ such that the nearest $\omega$ has $\Re[\omega]=u_{i}$. In our second step, we verified the hypotheses of part 2 of Proposition [10] so we have

$$
|z|^{2 n+2}=1-2(n+1) \Re[R]+(n+1)\left(\Gamma_{1}+\Gamma_{2} u_{i}\right)(1-\beta)^{2}+\mathcal{O}(1-\beta)^{\alpha+1} .
$$

Since $|z| \leq 1$, this implies that

$$
-\Re[R] \leq-\frac{\Gamma_{1}+\Gamma_{2} u_{i}}{2}(1-\beta)^{2}+\mathcal{O}(1-\beta)^{\alpha+1}
$$

and so from the definition of $R$ in Proposition 10 we have

$$
\begin{aligned}
-\Re\left[a_{n-1} \frac{\bar{\omega}-1}{n}+a_{n-2} \frac{\bar{\omega}^{2}-1}{n-1}\right. & \left.+\cdots+a_{0}(\omega-1)\right] \\
\leq & (1-\beta)-\frac{\Gamma_{1}+\Gamma_{2} u_{i}}{2}(1-\beta)^{2}+\mathcal{O}(1-\beta)^{\alpha+1} .
\end{aligned}
$$

Since $\Re(\bar{\omega})=u_{i}$, this inequality is also valid when $\omega$ is replaced by $\bar{\omega}$. Note that by Proposition 9 we have $\Re\left[a_{n-k}\right]=\mathcal{O}(1-\beta)^{3}$ for $k \geq 5$, so averaging these two inequalities gives us

$$
\begin{aligned}
\frac{\Re\left[a_{n-1}\right]}{n} \Re[1-\omega]+\cdots+ & \frac{\Re\left[a_{n-4}\right]}{n-3} \Re\left[1-\omega^{4}\right] \\
& \leq(1-\beta)-\frac{\Gamma_{1}+\Gamma_{2} u_{i}}{2}(1-\beta)^{2}+\mathcal{O}(1-\beta)^{\alpha+1} .
\end{aligned}
$$

Note that since $|\omega|=1$, then $\Re\left[\omega^{2}\right]=2 u_{i}^{2}-1, \Re\left[\omega^{3}\right]=4 u_{i}^{3}-3 u_{i}$ and $\Re\left[\omega^{4}\right]=$ $8 u_{i}^{4}-8 u_{i}^{2}+1$. Dividing inequality (3.13) by $1-u_{i}$, we get

$$
\begin{aligned}
\frac{\Re\left[a_{n-1}\right]}{n}+\frac{\Re\left[a_{n-2}\right]}{n-1}\left(2+2 u_{i}\right) & +\frac{\Re\left[a_{n-3}\right]}{n-2}\left(1+4 u_{i}+4 u_{i}^{2}\right)+\frac{\Re\left[a_{n-4}\right]}{n-3}\left(8 u_{i}^{2}+8 u_{i}^{3}\right) \\
& \leq \frac{1-\beta}{1-u_{i}}-\frac{\left(\Gamma_{1}+\Gamma_{2} u_{i}\right)(1-\beta)^{2}}{2\left(1-u_{i}\right)}+\mathcal{O}(1-\beta)^{\alpha+1} .
\end{aligned}
$$

Applying to this the linear transformation $\mathcal{T}$ defined in (2.3) and using the values computed in (2.4), we get an inequality of the form

$$
\begin{aligned}
\Re\left[a_{n-1}\right]+\Re\left[a_{n-2}\right]+c_{3} \Re\left[a_{n-3}\right]+c_{4} \Re\left[a_{n-4}\right] \\
\leq\left(n+n D_{1}+D_{2}\right)(1-\beta) \\
\quad-\left[\left(\Gamma_{1} / 2\right)\left(n+n D_{1}+D_{2}\right)+\left(\Gamma_{2} / 2\right)\left(n D_{1}+D_{2}\right)\right](1-\beta)^{2} \\
\quad+\mathcal{O}(1-\beta)^{\alpha+1},
\end{aligned}
$$

where $c_{3}=\mathcal{T}\left(1+4 u+4 u^{2}\right) /(n-2)$ and $c_{4}=\mathcal{T}\left(8 u^{2}+8 u^{3}\right) /(n-3)$. 
Define

$$
\begin{aligned}
Q=\left(-\Gamma_{1} / 2\right)\left(n+n D_{1}+D_{2}\right) & -\left(\Gamma_{2} / 2\right)\left(n D_{1}+D_{2}\right) \\
& -(n-1)(n-2)\left(1-c_{3}\right) D_{2}\left(1+D_{1}+D_{2} / n\right) .
\end{aligned}
$$

Recall from our second step that for all $n$ we have that $\Im\left[a_{n-1}\right]=\mathcal{O}(1-\beta)^{3 / 2}$, and that $\Re\left[a_{n-2}\right]=-(n-1) D_{2}(1-\beta)+\mathcal{O}(1-\beta)^{2}$, and that each $\left|\zeta_{j}-\beta\right|=$ $1+\left(D_{1}+D_{2} / n\right)(1-\beta)+\mathcal{O}(1-\beta)^{2}$. Then by part 2 of Lemma 13, we have

$$
\Re\left[a_{n-3}\right]+2 \Re\left[a_{n-4}\right]=-(n-1)(n-2) D_{2}\left(1+D_{1}+D_{2} / n\right)(1-\beta)^{2}+\mathcal{O}(1-\beta)^{3} .
$$

Adding $1-c_{3}$ times this to inequality (3.14) gives us

$$
\begin{aligned}
\Re\left[a_{n-1}\right]+\Re\left[a_{n-2}\right] & +\Re\left[a_{n-3}\right]+\left(2-2 c_{3}+c_{4}\right) \Re\left[a_{n-4}\right] \\
\leq & \left(n+n D_{1}+D_{2}\right)(1-\beta)+Q(1-\beta)^{2}+\mathcal{O}(1-\beta)^{\alpha+1} .
\end{aligned}
$$

Note that Lemma 11 implies that $c_{3}<1 / 2$ for $n \neq 3,4$, and 6 and that $c_{4} \geq 0$ for all $n$. Using the definition of $\mathcal{T}$ in (2.3), we calculate that for $n=4$ we have $c_{3}=3 / 2$ and $c_{4}=4$, and for $n=6$ we have $c_{3}=0.729$ and $c_{4}=0.972$. Thus for all $n \geq 4$ we have $1-2 c_{3}+c_{4}>0$. Note also that by our second step and Lemma 14 we have $\Re\left[a_{n-4}\right] \geq \delta(1-\beta)^{2}+\mathcal{O}(1-\beta)^{\alpha+1}$. Since $\delta=0$ except when $n=5$, and for $n=5$ we calculate $c_{3}=1 / 3$ and $c_{4}=2$, then

$$
\begin{aligned}
-\left(1-2 c_{3}+c_{4}\right) \Re\left[a_{n-4}\right] & \leq-\left(1-2 c_{3}+c_{4}\right) \delta(1-\beta)^{2}+\mathcal{O}(1-\beta)^{\alpha+1} \\
& =(-7 \delta / 3)(1-\beta)^{2}+\mathcal{O}(1-\beta)^{\alpha+1} .
\end{aligned}
$$

Adding this to equation (3.16) gives us

$$
\begin{aligned}
\Re\left[a_{n-1}+\right. & \left.a_{n-2}+a_{n-3}+a_{n-4}\right] \\
& \leq\left(n+n D_{1}+D_{2}\right)(1-\beta)+(Q-7 \delta / 3)(1-\beta)^{2}+\mathcal{O}(1-\beta)^{\alpha+1} .
\end{aligned}
$$

Let

$$
\begin{aligned}
& Q_{1}=-n(1-\beta)+a_{n-1}+a_{n-2}+a_{n-3}+a_{n-4}+a_{n-5} \quad \text { and } \\
& Q_{2}=n(n-1)(1-\beta)^{2} / 2-\left[(n-1) a_{n-1}+(n-2) a_{n-2}\right](1-\beta) .
\end{aligned}
$$

Recall from our first step that each $a_{n-k}=\mathcal{O}(1-\beta)$ so $Q_{1}=\mathcal{O}(1-\beta)$ and $Q_{2}=\mathcal{O}(1-\beta)^{2}$.

Now from our second step we know that $a_{n-k}=\mathcal{O}(1-\beta)^{\alpha}$ for $k \geq 3$, and from Proposition 9 we know that $a_{n-k}=\mathcal{O}(1-\beta)^{3}$ for $k \geq 6$, so

$$
\begin{array}{rl}
P^{\prime}(\beta)= & \beta^{n}+a_{n-1} \beta^{n-1}+\cdots+a_{0} \\
=1 & n(1-\beta)+\frac{n(n-1)}{2}(1-\beta)^{2}+a_{n-1}[1-(n-1)(1-\beta)] \\
& \quad+a_{n-2}[1-(n-2)(1-\beta)]+a_{n-3}+a_{n-4}+a_{n-5}+\mathcal{O}(1-\beta)^{\alpha+1} \\
=1 & +Q_{1}+Q_{2}+\mathcal{O}(1-\beta)^{\alpha+1} .
\end{array}
$$

Then $\left|P^{\prime}(\beta)\right|^{2}=P^{\prime}(\beta) \overline{P^{\prime}(\beta)}=1+2 \Re\left[Q_{1}\right]+2 \Re\left[Q_{2}\right]+\left|Q_{1}\right|^{2}+\mathcal{O}(1-\beta)^{\alpha+1}$. Note from our second step that each $\Im\left[a_{n-k}\right]=\mathcal{O}(1-\beta)^{\alpha}$ so $\Im\left[Q_{1}\right]=\mathcal{O}(1-\beta)^{\alpha}$. Then $\left(1+\Re\left[Q_{1}\right]+\Re\left[Q_{2}\right]\right)^{2}=\left|P^{\prime}(\beta)\right|^{2}+\mathcal{O}(1-\beta)^{\alpha+1}$ and so $\left|P^{\prime}(\beta)\right|=1+\Re\left[Q_{1}\right]+\Re\left[Q_{2}\right]+$ 
$\mathcal{O}(1-\beta)^{\alpha+1}$. Substituting the values of $Q_{1}$ and $Q_{2}$ and using the results of our second step gives us

$$
\begin{aligned}
\left|P^{\prime}(\beta)\right|=1 & -n(1-\beta)+\Re\left[a_{n-1}+a_{n-2}+a_{n-3}+a_{n-4}\right] \\
& +(n-1)\left[n / 2-n\left(1+D_{1}+D_{2}\right)+(n-2) D_{2}\right](1-\beta)^{2} \\
& +\mathcal{O}(1-\beta)^{\alpha+1} .
\end{aligned}
$$

Using the first line of inequality (3.7), then inequalities (3.18) and (3.17), we have

$$
\begin{aligned}
|P|_{\beta}^{n} \leq \mid & P^{\prime}(\beta) \mid \\
\leq 1 & +\left(n D_{1}+D_{2}\right)(1-\beta) \\
& +\left[Q-7 \delta / 3-(n-1)\left(n / 2+n D_{1}+2 D_{2}\right)\right](1-\beta)^{2} \\
& +\mathcal{O}(1-\beta)^{\alpha+1} .
\end{aligned}
$$

We now seek to compute the coefficient of $(1-\beta)^{2}$ in this inequality. Note first that from the definitions of $\Gamma_{1}$ and $\Gamma_{2}$ in Proposition 10 we have

$$
\begin{aligned}
-\frac{\Gamma_{1}}{2}\left(n+n D_{1}+D_{2}\right)- & \frac{\Gamma_{2}}{2}\left(n D_{1}+D_{2}\right) \\
= & -\frac{\Gamma_{1}+\Gamma_{2}}{2}\left(n+n D_{1}+D_{2}\right)+\frac{n \Gamma_{2}}{2} \\
= & {\left[\left(1+2 D_{1}\right) n-\left(\frac{1}{2}+2 D_{1}-2 D_{2}\right)\right]\left[\left(1+D_{1}\right) n+D_{2}\right] } \\
& \quad+n\left(1+D_{1}+D_{2}\right)\left[n D_{2}+\left(D_{1}-2 D_{2}\right)\right] .
\end{aligned}
$$

Now from the definition of $c_{3}$ (after inequality (3.14) ) combined with equalities (2.4) we have $(n-2) c_{3} D_{2}=-\left(n+1+D_{1}+3 n D_{1}+3 D_{2}\right)$ and so

$$
(n-2)\left(1-c_{3}\right) D_{2}=\left(1+3 D_{1}+D_{2}\right) n+\left(1+D_{1}+D_{2}\right) .
$$

Substituting these values into equation (3.15) and collecting like powers of $n$, we conclude that

$$
\begin{aligned}
Q=[ & \left.-D_{1}-D_{1}^{2}+D_{2}^{2}\right] n^{2}+\left[-\frac{1}{2}+\frac{1}{2} D_{1}+D_{1}^{2}-3 D_{2}^{2}\right] n \\
& +\left[1+2 D_{1}+\frac{1}{2} D_{2}+D_{1}^{2}+D_{1} D_{2}+2 D_{2}^{2}\right]+\left[D_{2}+D_{1} D_{2}+D_{2}^{2}\right] / n
\end{aligned}
$$

and so comparing this with the definition of $D$ in Theorem 1 , we see that

$$
Q-(n-1)\left(n / 2+n D_{1}+2 D_{2}\right)=n D+\frac{n(n-1)}{2}\left(D_{1}+D_{2} / n\right)^{2} .
$$

Substituting this into inequality (3.19), we have

$$
\begin{aligned}
|P|_{\beta}^{n} \leq 1 & +\left(n D_{1}+D_{2}\right)(1-\beta) \\
& +\left[n D+\frac{n(n-1)}{2}\left(D_{1}+D_{2} / n\right)^{2}-7 \delta / 3\right](1-\beta)^{2}+\mathcal{O}(1-\beta)^{\alpha+1} \\
= & {\left[1+\left(D_{1}+D_{2} / n\right)(1-\beta)+\left(D-\frac{7 \delta}{3 n}\right)(1-\beta)^{2}\right]^{n}+\mathcal{O}(1-\beta)^{\alpha+1} . }
\end{aligned}
$$


Note that (from the definitions of $\delta$ in Lemma 14 and $\Delta$ in Theorem 10) for all $n$ we have $\Delta=-7 \delta /(3 n)$, and so

$$
|P|_{\beta} \leq 1+\left(D_{1}+D_{2} / n\right)(1-\beta)+(D+\Delta)(1-\beta)^{2}+\mathcal{O}(1-\beta)^{\alpha+1} .
$$

This completes the proof of Proposition 5

\section{Proof of Proposition 6}

This proof parallels the proof of [8, Theorem 2]. We begin by letting

$$
u=\frac{-i \sqrt{15}}{15}(1-\beta)^{1 / 2}-\frac{6}{10}(1-\beta)+\frac{i \sqrt{15}}{300}(1-\beta)^{3 / 2}-\frac{33}{600}(1-\beta)^{2}
$$

and

$$
v=\frac{4 i \sqrt{15}}{15}(1-\beta)^{1 / 2}-\frac{1}{10}(1-\beta)+\frac{46 i \sqrt{15}}{300}(1-\beta)^{3 / 2}+\frac{532}{600}(1-\beta)^{2} .
$$

Let $P^{\prime}(z)=(z-u)^{4}(z-v)$ and let $P(z)=\int_{\beta}^{z} P^{\prime}(t) d t$. Note that $u-\beta=$ $-1+u+(1-\beta)$ so

$$
\begin{aligned}
|u-\beta|^{2}= & {\left[-1+(4 / 10)(1-\beta)-(33 / 600)(1-\beta)^{2}\right]^{2} } \\
& +\left[(-\sqrt{15} / 15)(1-\beta)^{1 / 2}+(\sqrt{15} / 300)(1-\beta)^{3 / 2}\right]^{2} \\
= & 1-(11 / 15)(1-\beta)+(79 / 300)(1-\beta)^{2}+\mathcal{O}(1-\beta)^{3}
\end{aligned}
$$

and $v-\beta=-1+v+(1-\beta)$ so

$$
\begin{aligned}
|v-\beta|^{2}=[ & \left.-1+(9 / 10)(1-\beta)+(532 / 600)(1-\beta)^{2}\right]^{2} \\
& +\left[(4 \sqrt{15} / 15)(1-\beta)^{1 / 2}+(46 \sqrt{15} / 300)(1-\beta)^{3 / 2}\right]^{2} \\
= & 1-(11 / 15)(1-\beta)+(79 / 300)(1-\beta)^{2}+\mathcal{O}(1-\beta)^{3} .
\end{aligned}
$$

Now

$$
\begin{aligned}
{[1-(11 / 30)(1-\beta)+} & \left.(29 / 450)(1-\beta)^{2}\right]^{2} \\
& =1-(11 / 15)(1-\beta)+(79 / 300)(1-\beta)^{2}+\mathcal{O}(1-\beta)^{3},
\end{aligned}
$$

and so we have

$$
\begin{aligned}
|P|_{\beta} & =\min \{|u-\beta|,|v-\beta|\} \\
& =1-(11 / 30)(1-\beta)+(29 / 450)(1-\beta)^{2}+\mathcal{O}(1-\beta)^{3} .
\end{aligned}
$$

By definition $P$ is of degree 6 and $P(\beta)=0$. Thus to verify that $P \in S(6, \beta)$ we need only show that all the roots of $P$ remain in the closed unit disk when $\beta$ is sufficiently close to 1 . Now

$$
\begin{aligned}
& u^{2}=(-1 / 15)(1-\beta)+(2 i \sqrt{15} / 25)(1-\beta)^{3 / 2}+\mathcal{O}(1-\beta)^{2}, \\
& u^{3}=(i \sqrt{15} / 225)(1-\beta)^{3 / 2}+\mathcal{O}(1-\beta)^{2}, \quad \text { and } \\
& u^{4}=\mathcal{O}(1-\beta)^{2},
\end{aligned}
$$


so writing $P^{\prime}(z)=z^{5}+a_{4} z^{4}+\cdots+a_{0}$, we calculate that

$$
\begin{aligned}
a_{4} & =-(4 u+v)=(5 / 2)(1-\beta)-(i \sqrt{15} / 6)(1-\beta)^{3 / 2}-(2 / 3)(1-\beta)^{2} \\
a_{3} & =u(6 u+4 v) \\
& =(2 / 3)(1-\beta)-(2 i \sqrt{15} / 15)(1-\beta)^{3 / 2}+3(1-\beta)^{2}+\mathcal{O}(1-\beta)^{5 / 2} \\
a_{2} & =-u^{2}(4 u+6 v)=(4 i \sqrt{15} / 45)(1-\beta)^{3 / 2}+(7 / 5)(1-\beta)^{2}+\mathcal{O}(1-\beta)^{5 / 2} \\
a_{1} & =u^{3}(u+4 v)=(-1 / 15)(1-\beta)^{2}+\mathcal{O}(1-\beta)^{5 / 2} \\
a_{0} & =-u^{4} v=\mathcal{O}(1-\beta)^{5 / 2} .
\end{aligned}
$$

Recall from the values computed at the beginning of section 2 that for $n=5$ we have $\alpha=3 / 2, u_{1}=-1 / 2, u_{2}=-1, D_{1}=-1 / 3$ and $D_{2}=-1 / 6$. Note that in part 2 of Proposition 10 the values of the $a_{k}$ 's computed above satisfy the hypotheses, and that $\Gamma_{2}=-5 / 6$ and $\Gamma_{1}=-13 / 6$.

Let us apply part 2 of Proposition 10 to the case $\omega=-1$. Note that $\Re[\omega]=u_{2}$ and $\Gamma_{1}+\Gamma_{2} u_{2}=-4 / 3$. Since $\omega=-1$ we have

$$
R=(1-\beta)-(2 / 5) a_{4}-(2 / 3) a_{2}-2 a_{0},
$$

and so

$$
\begin{aligned}
\Re[R]= & (1-\beta)-(2 / 5)\left[(5 / 2)(1-\beta)-(2 / 3)(1-\beta)^{2}\right] \\
& -(2 / 3)(7 / 5)(1-\beta)^{2}+\mathcal{O}(1-\beta)^{5 / 2} \\
= & (-2 / 3)(1-\beta)^{2}+\mathcal{O}(1-\beta)^{5 / 2} .
\end{aligned}
$$

Thus by part 2 of Proposition 10 we have

$$
\begin{aligned}
|z|^{12} & =1-12(-2 / 3)(1-\beta)^{2}+6(-4 / 3)(1-\beta)^{2}+\mathcal{O}(1-\beta)^{5 / 2} \\
& =1+\mathcal{O}(1-\beta)^{5 / 2}
\end{aligned}
$$

and so $|z|=1+\mathcal{O}(1-\beta)^{5 / 2}$.

Let us now apply part 2 of Proposition [10 to the case $\omega=(1 / 2)(-1 \pm i \sqrt{3})$. Note that $\Re[\omega]=u_{1}$ and $\Gamma_{1}+\Gamma_{2} u_{1}=-7 / 4$. Now

$$
\begin{aligned}
R=(1-\beta)+\left(a_{4} / 10\right)(-3 \mp i \sqrt{3})+ & \left(a_{3} / 8\right)(-3 \pm i \sqrt{3}) \\
& +\left(a_{1} / 4\right)(-3 \mp i \sqrt{3})+\left(a_{0} / 2\right)(-3 \pm i \sqrt{3})
\end{aligned}
$$

SO

$$
\begin{aligned}
& \Re[R]=(1-\beta)-(3 / 10)\left[(5 / 2)(1-\beta)-(2 / 3)(1-\beta)^{2}\right] \\
& \pm(\sqrt{3} / 10)(-\sqrt{15} / 6)(1-\beta)^{3 / 2}-(3 / 8)\left[(2 / 3)(1-\beta)+3(1-\beta)^{2}\right] \\
& \mp(\sqrt{3} / 8)(-2 \sqrt{15} / 15)(1-\beta)^{3 / 2}-(3 / 4)(-1 / 15)(1-\beta)^{2}+\mathcal{O}(1-\beta)^{5 / 2} \\
&=(-7 / 8)(1-\beta)^{2}+\mathcal{O}(1-\beta)^{5 / 2} .
\end{aligned}
$$

Thus by part 2 of Proposition 10 we have

$$
\begin{aligned}
|z|^{12} & =1-12(-7 / 8)(1-\beta)^{2}+6(-7 / 4)(1-\beta)^{2}+\mathcal{O}(1-\beta)^{5 / 2} \\
& =1+\mathcal{O}(1-\beta)^{5 / 2}
\end{aligned}
$$

so $|z|=1+\mathcal{O}(1-\beta)^{5 / 2}$. 
Finally, let us apply part 1 of Proposition 10 with $r=1$ to the case $\omega=$ $(1 / 2)(1 \pm i \sqrt{3})$. Note that

$$
R=(1-\beta)+\left(a_{4} / 10\right)(-1 \mp i \sqrt{3})+\left(a_{3} / 8\right)(-3 \mp i \sqrt{3})+\mathcal{O}(1-\beta)^{3 / 2}
$$

so

$$
\begin{aligned}
\Re[R] & =(1-\beta)+(-1 / 10)(5 / 2)(1-\beta)+(-3 / 8)(2 / 3)(1-\beta)+\mathcal{O}(1-\beta)^{3 / 2} \\
& =(1 / 2)(1-\beta)+\mathcal{O}(1-\beta)^{3 / 2} .
\end{aligned}
$$

Thus by part 1 of Proposition 10 we have $|z|^{2}=1-(1-\beta)+\mathcal{O}(1-\beta)^{3 / 2}$ and so $|z|=1-(1 / 2)(1-\beta)+\mathcal{O}(1-\beta)^{3 / 2}$.

At this stage, we know that $|P|_{\beta}=1-(11 / 30)(1-\beta)+(29 / 450)(1-\beta)^{2}+$ $\mathcal{O}(1-\beta)^{3}$ and that if $\beta$ is sufficiently close to 1 , then all roots $z$ of $P$ have $|z| \leq$ $1+\mathcal{O}(1-\beta)^{5 / 2}$. Since the roots of $P$ approach the roots of $z^{6}-1$, then the non- $\beta$ roots of $P$ are bounded away from $\beta$. Thus by Lemma 12, there is a polynomial $Q \in S(6, \beta)$ with $|Q|_{\beta}=1-(11 / 30)(1-\beta)+(29 / 450)(1-\beta)^{2}+\mathcal{O}(1-\beta)^{5 / 2}$. This completes the proof of Proposition [6]

\section{Proof of Proposition 7}

Let $b_{1}=1+D_{1}+D_{2} / n$, let $b_{2}=(n-1) D_{2}$, and let $z_{0}=-b_{1}(1-\beta)-D(1-\beta)^{2}$. Then $z_{0}-\beta=-1+\left(1-b_{1}\right)(1-\beta)-D(1-\beta)^{2}$, and (for $\beta$ near 1$)$ this is real and negative so $\left|z_{0}-\beta\right|=1+\left(D_{1}+D_{2} / n\right)(1-\beta)+D(1-\beta)^{2}$.

Now let $x$ be a real constant, depending only on $n$ (and to be determined later), and let

$$
\begin{aligned}
q(z)=z^{2}+\left[\left(b_{2}+2 b_{1}\right)(1-\beta)\right. & \left.-2 x(1-\beta)^{2}\right] z \\
+ & {\left[-b_{2}(1-\beta)+\left(b_{1}^{2}+b_{2}+2 D+2 x\right)(1-\beta)^{2}\right] . }
\end{aligned}
$$

Now by part 4 of Lemma 8 we have $D_{2}<0$ and so $b_{2}<0$. Since the discriminant of $q(z)$ is $4 b_{2}(1-\beta)+\mathcal{O}(1-\beta)^{2}$, then (for $\beta$ near 1 ) the roots of $q$ are complex conjugates. If we denote these roots by $z_{1}$ and $\bar{z}_{1}$, then by writing $\beta=1-(1-\beta)$ we have

$$
\begin{aligned}
\left|z_{1}-\beta\right|^{2} & =\left(z_{1}-\beta\right)\left(\bar{z}_{1}-\beta\right)=q(\beta) \\
& =1+\left(2 b_{1}-2\right)(1-\beta)+\left(1-2 b_{1}+b_{1}^{2}+2 D\right)(1-\beta)^{2}+\mathcal{O}(1-\beta)^{3} \\
& =\left[1+\left(b_{1}-1\right)(1-\beta)+D(1-\beta)^{2}\right]^{2}+\mathcal{O}(1-\beta)^{3},
\end{aligned}
$$

so $\left|z_{1}-\beta\right|=1+\left(D_{1}+D_{2} / n\right)(1-\beta)+D(1-\beta)^{2}+\mathcal{O}(1-\beta)^{3}$.

Let $P^{\prime}(z)=\left(z-z_{0}\right)^{n-2} q(z)$ and $P(z)=\int_{\beta}^{z} P^{\prime}(t) d t$, so

$$
|P|_{\beta}=1+\left(D_{1}+D_{2} / n\right)(1-\beta)+D(1-\beta)^{2}+\mathcal{O}(1-\beta)^{3} .
$$

Now $z_{0}=\mathcal{O}(1-\beta)$, so

$$
\begin{gathered}
\left(z-z_{0}\right)^{n-2}=z^{n-2}-(n-2) z_{0} z^{n-3}+\left(\begin{array}{c}
n-2 \\
2
\end{array}\right) z_{0}^{2} z^{n-4}+\mathcal{O}(1-\beta)^{3} \\
=z^{n-2}+(n-2)\left[b_{1}(1-\beta)+D(1-\beta)^{2}\right] z^{n-3} \\
\quad+\left(\begin{array}{c}
n-2 \\
2
\end{array}\right) b_{1}^{2}(1-\beta)^{2} z^{n-4}+\mathcal{O}(1-\beta)^{3}
\end{gathered}
$$


Then letting $t_{1}=\left(n^{2}-n\right) b_{1}^{2} / 2+(n-2) b_{1} b_{2}+b_{2}$ we have

$$
\begin{aligned}
P^{\prime}(z)= & \left(z-z_{0}\right)^{n-2} q(z) \\
=z^{n} & +\left[\left(n b_{1}+b_{2}\right)(1-\beta)+(n D-2 D-2 x)(1-\beta)^{2}\right] z^{n-1} \\
& +\left[-b_{2}(1-\beta)+\left(t_{1}+2 D+2 x\right)(1-\beta)^{2}\right] z^{n-2} \\
& -(n-2) b_{1} b_{2}(1-\beta)^{2} z^{n-3}+\mathcal{O}(1-\beta)^{3} .
\end{aligned}
$$

Note that by its definition, $P$ is a polynomial of degree $n+1$ and $P(\beta)=0$. Thus to show that $P \in S(n+1, \beta)$ it will suffice to show that all roots of $P$ remain in the unit disk when $\beta$ is sufficiently close to 1 .

Let $\omega \neq 1$ be an $(n+1)$ th root of 1 , let $u=\Re[\omega]$ and note that since $|\omega|=1$, then $\Re\left[\omega^{2}\right]=2 u^{2}-1, \Re\left[\omega^{3}\right]=4 u^{3}-3 u$, and $\omega^{n-k}=\bar{\omega}^{k+1}$. Substituting the coefficients of equation (5.1) into the formula for $R$ in Proposition 10, we have

$$
\begin{aligned}
R=(1-\beta)+\left(n b_{1}+b_{2}\right)(1-\beta)(\bar{\omega}-1) / n & \\
& -b_{2}(1-\beta)\left(\bar{\omega}^{2}-1\right) /(n-1)+\mathcal{O}(1-\beta)^{2} .
\end{aligned}
$$

Substituting the values of $b_{1}$ and $b_{2}$ into this formula, we see by part 1 of Proposition 10 with $r=1$ that

$$
|z|^{2}=1-2(1-\beta)\left[1+\left(1+D_{1}+D_{2}\right)(u-1)-D_{2}\left(2 u^{2}-2\right)\right]+\mathcal{O}(1-\beta)^{2} .
$$

Recall from part 4 of Lemma 8 that $D_{2}<0$, so the quantity in square brackets is quadratic in $u$ with positive leading coefficient. By elementary calculus, its minimum (over all real numbers) occurs when $1+D_{1}+D_{2}-4 D_{2} u=0$, which happens when $u=\left(1+D_{1}+D_{2}\right) /\left(4 D_{2}\right)=\left(u_{1}+u_{2}\right) / 2$, which is between $u_{1}$ and $u_{2}$. Now $u_{1}$ and $u_{2}$ are (by definition) the real parts of adjacent $(n+1)$ th roots of 1 , so there are no possible values of $u$ between $u_{1}$ and $u_{2}$, so the minimum (over all possible values of $u$ ) must occur at either $u_{1}$ or $u_{2}$. From part 7 of Lemma 8 we see that at these values the quantity in square brackets is 0 , and so the minimum value of the quantity in square brackets is 0 . Thus for $\Re[\omega] \neq u_{i}$ the quantity in square brackets is positive, so for these values of $\omega$ and for $\beta$ sufficiently close to 1 we have $|z|<1$, and so these roots remain in the unit disk.

Thus we need only concern ourselves with the case $\Re[\omega]=u_{i}$. In this case, by part 2 of Proposition 10 we have

$$
|z|^{2 n+2}=1-2(n+1) \Re[R]+(n+1)\left(\Gamma_{1}+\Gamma_{2} u_{i}\right)(1-\beta)^{2}+\mathcal{O}(1-\beta)^{\alpha+1} .
$$

To get $P \in S(n+1, \beta)$ we will seek a value of $x$ so that $|z|=1+\mathcal{O}(1-\beta)^{\alpha+1}$, so we will need

$$
\Re[R]-(1 / 2)\left(\Gamma_{1}+\Gamma_{2} u_{i}\right)(1-\beta)^{2}=\mathcal{O}(1-\beta)^{\alpha+1}
$$

for both $i=1$ and $i=2$.

Substituting the coefficients of equation (5.1) into the formula for $R$ in Proposition 10, we have

$$
\begin{aligned}
R=(1 & -\beta)+\left[\left(n b_{1}+b_{2}\right)(1-\beta)+(n D-2 D-2 x)(1-\beta)^{2}\right](\bar{\omega}-1) / n \\
& +\left[-b_{2}(1-\beta)+\left(t_{1}+2 D+2 x\right)(1-\beta)^{2}\right]\left(\bar{\omega}^{2}-1\right) /(n-1) \\
& -(n-2) b_{1} b_{2}(1-\beta)^{2}\left(\bar{\omega}^{3}-1\right) /(n-2)+\mathcal{O}(1-\beta)^{3} .
\end{aligned}
$$


Taking the real parts of equation (5.3) and collecting like powers of $(1-\beta)$ gives us

$$
\begin{aligned}
\Re[R]=[1+ & \left.\left(n b_{1}+b_{2}\right)\left(u_{i}-1\right) / n-b_{2}\left(2 u_{i}^{2}-2\right) /(n-1)\right](1-\beta) \\
+ & {\left[(n D-2 D-2 x)\left(u_{i}-1\right) / n+\left(t_{1}+2 D+2 x\right)\left(2 u_{i}^{2}-2\right) /(n-1)\right.} \\
& \left.-b_{1} b_{2}\left(4 u_{i}^{3}-3 u_{i}-1\right)\right](1-\beta)^{2}+\mathcal{O}(1-\beta)^{3} .
\end{aligned}
$$

Substituting the values of $b_{1}$ and $b_{2}$ into this formula, we see from part 7 of Lemma 8 that the coefficient of $(1-\beta)$ in $\Re[R]$ is zero, so to satisfy equation (5.2) we need only find a value of $x$ such that the coefficient of $(1-\beta)^{2}$ in equation (5.2) is 0 . We divide this coefficient by $u_{i}-1$ and denote the result by $Z_{i}$, so

$$
\begin{aligned}
& Z_{i}=(n D-2 D-2 x) / n+\left(t_{1}+2 D+2 x\right)\left(2 u_{i}+2\right) /(n-1) \\
& -(n-1) D_{2}\left(1+D_{1}+D_{2} / n\right)\left(4 u_{i}^{2}+4 u_{i}+1\right)+(1 / 2)\left(\Gamma_{1}+\Gamma_{2} u_{i}\right) /\left(1-u_{i}\right) .
\end{aligned}
$$

Note that the coefficient of $x$ in $Z_{i}$ is $-2 / n+\left(4 u_{i}+4\right) /(n-1)$, which is non-zero by part 3 of Lemma 8 , so each equation $Z_{i}=0$ has a solution for $x$. To show that these solutions are identical, we will show that $Z_{1}$ and $Z_{2}$ (considered as linear expressions in the variable $x$ ) are scalar multiples of each other.

To see this, we eliminate $x$ by applying the transformation $\mathcal{T}$ defined in equation (2.3). Since in equation (3.14) we defined $c_{3}=\mathcal{T}\left(1+4 u+4 u^{2}\right) /(n-2)$, then from equations (2.4) we see that

$$
\begin{aligned}
\mathcal{T}\left(Z_{i}\right)=n D+t_{1}-(n-1) & (n-2) c_{3} D_{2}\left(1+D_{1}+D_{2} / n\right) \\
& +\left(\Gamma_{1} / 2\right)\left(n+n D_{1}+D_{2}\right)+\left(\Gamma_{2} / 2\right)\left(n D_{1}+D_{2}\right) .
\end{aligned}
$$

Comparing this to the value of $Q$ defined in equation (3.15), we see that

$$
\mathcal{T}\left(Z_{i}\right)=n D+t_{1}-Q-(n-1)(n-2) D_{2}\left(1+D_{1}+D_{2} / n\right) .
$$

Note that by equation (3.21) we have

$$
Q=n D+\frac{n(n-1)}{2}\left(D_{1}+D_{2} / n\right)^{2}+(n-1)\left(n / 2+n D_{1}+2 D_{2}\right) .
$$

Substituting the values of $b_{1}$ and $b_{2}$ into our definition of $t_{1}$ gives us

$$
t_{1}=(n-1)\left[(n / 2)\left(1+D_{1}+D_{2} / n\right)^{2}+(n-2) D_{2}\left(1+D_{1}+D_{2} / n\right)+D_{2}\right]
$$

and so $Q-t_{1}=n D-(n-1)(n-2) D_{2}\left(1+D_{1}+D_{2} / n\right)$. Substituting this into equation (5.6) gives us $\mathcal{T}\left(Z_{i}\right)=0$. Since $\mathcal{T}\left(Z_{i}\right)$ is a linear combination of $Z_{1}$ and $Z_{2}$, this implies that $Z_{1}$ and $Z_{2}$ (considered as polynomials in $x$ ) are scalar multiples of one another, and so there is a single value of $x$ that satisfies equation (5.2) for both $i=1$ and $i=2$.

Using this value of $x$, we have now constructed a real polynomial $P$ with

$$
|P|_{\beta}=1+\left(D_{1}+D_{2} / n\right)(1-\beta)+D(1-\beta)^{2}+\mathcal{O}(1-\beta)^{3}
$$

and such that all roots $z$ of $P$ have $|z| \leq 1+\mathcal{O}(1-\beta)^{\alpha+1}$. Since the roots of $P$ approach the roots of $z^{n+1}-1$, then the non- $\beta$ roots of $P$ are bounded away from $\beta$. Thus by Lemma 12, there is a real polynomial $Q \in S(n+1, \beta)$ with

$$
|Q|_{\beta}=1+\left(D_{1}+D_{2} / n\right)(1-\beta)+D(1-\beta)^{2}+\mathcal{O}(1-\beta)^{\alpha+1} .
$$

This finishes the proof of Proposition [7 


\section{REFERENCES}

[1] B. D. Bojanov, Q. I. Rahman and J. Szynal, On a conjecture of Sendov about the critical points of a polynomial, Math. Z. 190 (1985), 281-285. MR.0797543 (86j:30010)

[2] _ On a conjecture about the critical points of a polynomial, Delay Equations, Approximation and Application, Birkhäuser, Basel, 1985, 83-93. MR 0899090 (88e:30013)

[3] J. Borcea, The Sendov conjecture for polynomials with at most seven distinct zeros, Analysis 16 (1996), 137-159. MR1397576 (97g:30006)

[4] J. E. Brown and G. Xiang, Proof of the Sendov conjecture for polynomials of degree at most eight, J. Math. Anal. Appl. 232 (1999), 272-292. MR1683144 (2001b:30007)

[5] M. Marden, Geometry of Polynomials, 3rd Ed., American Mathematical Society, Providence, 1989. MR0225972 (37:1562)

[6] M. J. Miller, Maximal polynomials and the Ilieff-Sendov conjecture, Trans. Amer. Math. Soc. 321 (1990), 285-303. MR0965744 (90m:30007)

[7] _ On Sendov's conjecture for roots near the unit circle, J. Math. Anal. Appl. 175 (1993), 632-639. MR1219199 (94h:30003)

[8] _ Some maximal polynomials must be nonreal, J. Math. Anal. Appl. 214 (1997), 283291. MR 1645480 (99h:41010)

[9] Q. I. Rahman, On the zeros of a polynomial and its derivative, Pacific J. Math. 41 (1972), 525-528. MR0308374 (46:7488)

[10] Z. Rubinstein, On a problem of Ilyeff, Pacific J. Math. 26 (1968), 159-161. MR0237753 (38:6034)

[11] J. V. Uspensky, Theory of Equations, McGraw-Hill, New York, 1948.

[12] V. Vâjâitu and A. Zaharescu, Ilyeff's conjecture on a corona, Bull. London Math. Soc. 25 (1993), 49-54. MR1190363 (94h:30004)

Department of Mathematics, Le Moyne College, Syracuse, New York 13214

E-mail address: millermj@mail. lemoyne.edu 